The following manuscript is a pre-print. This article has not yet been peer reviewed.

Augusteijn, H.E.M., van Aert, R.C.M., \& van Assen, M.A.L.M. (2021). Posterior Probabilities of Effect Sizes and Heterogeneity in Meta-Analysis: An Intuitive Approach of Dealing with Publication Bias. https://doi.org/10.17605/OSF.IO/98DRQ

Feedback, suggestion, comments and remarks are more than welcome. They can be send to h.e.m.augusteijn@ tilburguniversity.edu.

Thank you,

Hilde, Robbie and Marcel 


\title{
Posterior Probabilities of Effect Sizes and Heterogeneity in Meta-Analysis: An Intuitive Approach of Dealing with Publication Bias
}

\author{
H.E.M. Augusteijn \\ R.C.M. van Aert \\ M.A.L.M. van Assen
}

Publication bias remains to be a great challenge when conducting a metaanalysis. It may result in overestimated effect sizes, increased frequency of false positives, and over- or underestimation of the effect size heterogeneity parameter. A new method is introduced, Bayesian Meta-Analytic Snapshot (BMAS), which evaluates both effect size and its heterogeneity and corrects for potential publication bias. It evaluates the probability of the true effect size being zero, small, medium or large, and the probability of true heterogeneity being zero, small, medium or large. This approach, which provides an intuitive evaluation of uncertainty in the evaluation of effect size and heterogeneity, is illustrated with a real-data example, a simulation study, and a Shiny web application of BMAS.

Keywords: meta-analysis, effect size, heterogeneity, publication bias, Bayesian statistics, posterior probabilities

Meta-analysis has become increasingly popular when trying to summarize the results available within a research field or regarding a specific research question. It allows us to combine the results of multiple studies and provides us with an estimate of the underlying effect size, and the variation thereof, called heterogeneity. This synthesis of research results is particularly relevant, since the number of published articles has also increased exponentially over the years (Bornmann \& Mutz, 2015). Meta-analysis may help us to get an overview of the large number of articles and is often considered to provide valuable information for policymakers (Cordray \& Morphy, 2009).

However, most meta-analyses depend (mainly) upon research results available in the published literature. This is problematic when the published literature is not representative of the research conducted on a specific topic. Multiple research fields provide evidence that there is preference in submission and publication of research findings based on the nature and direction of 
the research results (Cooper, DeNeve \& Charlton, 1997; Fanelli, 2012; Franco, Simonovits \& Malhotra, 2016). That is, statistically significant results are more likely to be published and are overrepresented within the published literature of many research fields. This bias is known under multiple names, such as publication bias and the file drawer problem. It has been a concern for a long time, dating back to at least the $18^{\text {th }}$ century (Dickersin, 2005).

In a research field where publication bias is present, statistically significant studies will be overrepresented and these generally have larger effect sizes than non-significant studies. Summarizing this biased sample of studies will often result in a overestimation of effect size, and a high probability of a false positive (or Type I error) if the null-hypothesis of a zero true effect size is actually true (e.g. Borenstein, Hedges, Higgins, \& Rothstein, 2009; Nuijten, van Assen, Veldkamp \& Wicherts, 2015). Conventional meta-analysis methods do not provide a correction for publication bias, but many publication bias correction methods have been developed over the years. Frequentist methods are, for example, trim-and-fill (Duval \& Tweedie, 2000), selection models (e.g., Hedges \& Vevea, 2005), PET-PEESE (Stanley \& Doucouliagos, 2014), p-curve (Simonsohn, Nelson, \& Simmons, 2014), p-uniform (van Assen, van Aert \& Wicherts, 2015), and p-uniform* (van Aert \& van Assen, 2021). Bayesian methods have been developed as well, such as a Bayesian model averaging method (Guan \& Vandekerckhove, 2016) and the Bayesian fill-inmeta-analysis method, named BALM (Du, Liu \& Wang, 2017).

All publication bias correction methods have different assumptions that need to be met, and none of these methods perform well under all circumstances (e.g. van Aert, Wicherts, van Assen, 2016; Stanley, 2017; Carter, Schönbrodt, Gervais \& Hilgard, 2019). For example, in realistic situations, selection models need a large amount of data, PET-PEESE needs a large variation in study sample sizes, and most methods, including $p$-curve and $p$-uniform, perform poorly in the presence of heterogeneity (McShane, Böckenholt \& Hansen, 2016; Carter et al., 2019). So far, the performance of the Bayesian meta-analysis methods has not been evaluated in the same extensive way as the frequentist methods, by others than the original authors. Furthermore, none of these methods, frequentist or Bayesian, have been designed to correct for questionable research practices or $p$-hacking, that is, the opportunistic use of design and analysis choices aimed at achieving a statistically significant p-value. Evidence suggests these methods may not perform well in its presence (van Aert et al., 2016; Friese \& Frankenbach, 2020). In the simulation studies by Carter et al. (2019), questionable research practices lead to overestimation 
for some methods, and underestimation and power loss for others. Conducting a sensitivity analysis, by applying multiple methods and comparing their results, is therefore relevant, but only if these methods can be expected to perform reasonably well for the characteristics of the dataset in question (Carter et al., 2019). Applying all available methods, even when some cannot be expected to perform well, will only provide more noise. Which methods to apply is not straightforward: which method performs well in which conditions is still a subject of research and researchers will always need to make assumptions about unknown and unobservable characteristics of their data.

This paper develops a new method, Bayesian Meta-Analytic Snapshot (BMAS), which evaluates both the effect size and heterogeneity of effect size and corrects for potential publication bias. The method considers the probability of four levels of effect size (no effect, a small, medium or large effect), and four levels of heterogeneity (no heterogeneity, small, medium or large heterogeneity). The probability of each of these 16 underlying data generating models (4 (effect size) $\times 4$ (heterogeneity)) depends on the data that is available and the prior believes of the researcher. This method uses Bayes theorem to determine these probabilities.

Three versions of this method are developed, differing in assumptions on publication bias and the treatment of statistically non-significant effect sizes. The first version, named 'Uncorrected BMAS', assumes there is no publication bias, that is, all studies are equally likely to be published. If these assumptions are met, this method is highly efficient. This first version is comparable to random effects meta-analysis, but only considers 16 models, instead of a potentially infinite number of models, and evaluates models in a Bayesian way. One major advantage of Bayesian model testing over frequentist Null Hypothesis Significance Testing is that evidence may be quantified for any hypothesis, and not only one (the alternative) hypothesis (Tendeiro, \& Kiers, 2019).

The second version, named 'BMAS', assumes that there may be publication bias, and makes a distinction between statistically significant and non-significant results: non-significant results are less likely to be published compared to those that are statistically significant, but it assumes that there is no difference in publication probability within the non-significant effect sizes. This version of the method is comparable to the common implementation of selection model methods such as the one by Hedges and Vevea (2005) that only treats significant and nonsignificant effect sizes differently. 


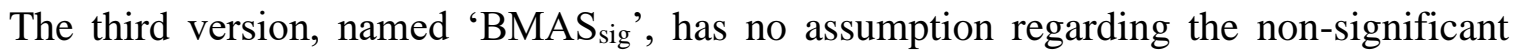
effect sizes, since it only takes the published studies into account. It has the least assumptions, but is also the least efficient, particularly if publication bias is not extreme and true effect size is small; in that context relatively many non-significant effect sizes are present, and BMAS ${ }_{\text {sig }}$ does not use these studies. BMAS $\mathrm{Big}_{\mathrm{sig}}$ is most comparable to $p$-uniform (van Assen et al., 2015) and $p$-curve (Simonsohn et al., 2014), which also make use of the distribution of statistically significant studies.

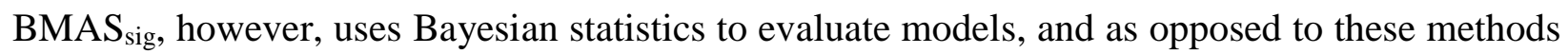
also evaluates heterogeneity of effect size.

The BMAS methods answer the meta-analytic question of the probability of the true effect size and does so in an intuitive way. It provides an easy and natural interpretation of the effect size and heterogeneity, shows whether there is sufficient data available to distinct between the different models, and shows the impact of potential publication bias, by comparing the corrected version(s) of the method with the uncorrected version.

\section{Meta-analytical methods}

In the presence of publication bias, conventional meta-analysis methods do not provide correct estimates, since they do not correct for this bias. They result in overestimations of the effect size (Lane \& Dunlap, 1978; Nuijten et al., 2015; Terrin, Schmid, Lau \& Olkin, 2003) and over- or under estimation of heterogeneity (Jackson, 2006; 2007; Augusteijn, van Aert \& van Assen, 2019). Many methods have been developed, aiming at correcting for publication bias, but, as previously discussed, so far no method performs well in all circumstances. The aim of the current project is to evaluate the performance of a new method, BMAS. In the next section, we will first provide a brief summary of random-effects meta-analysis, since its results will be used as baseline in the simulation studies.

\section{Random effects meta-analysis}

The random-effects model assumes that the primary studies included in the meta-analysis may originate from different underlying true effect sizes, that is, it assumes that there is heterogeneity in the true effect sizes. The aim of the random effects model is to model these differences, while still providing a meaningful average true effect size estimate (Jackson, 2006). The random effects model assumes that each observed effect size of a primary study $\left(Y_{i}\right)$ stems from an underlying grand mean, the true average effect size $(\mu)$, the deviation of the study's true effect size from the grand mean $\left(\zeta_{\mathrm{i}}\right)$ and the study's sampling error $\left(\varepsilon_{\mathrm{i}}\right)$ : 


$$
Y_{i}=\mu+\zeta_{i}+\varepsilon_{i}
$$

Both $\zeta_{\mathrm{i}}$ and $\varepsilon_{\mathrm{i}}$ are assumed to be normally distributed variables. The variance of the sampling error $\left(\varepsilon_{i}\right)$ is often referred to as the within-study variance $\left(\sigma_{i}^{2}\right)$, while the variance of the deviation from the grand mean $\left(\zeta_{\mathrm{i}}\right)$ is known as the between-study variance $\left(\tau^{2}\right)$. The variance of each study's effect size in the random effects model is the sum of these within- and between-study variances:

$$
\operatorname{Var}\left(Y_{i}\right)=\tau^{2}+\sigma_{i}^{2}
$$

$\sigma_{i}^{2}$ is a function of the population variance and the study sample size. In practice it is estimated and then assumed to be known.

Multiple methods can be used to estimate the between study variance, $\tau^{2}$. Even though the DerSimonian and Laird method (DerSimonian, Laird, 1986) is often outperformed by other methods (e.g. Restricted Maximum Likelihood (REML) and the Paule-Mandel method, see Langan, Higgins \& Simmonds, 2017) it is the most used and most commonly implemented estimator of the between study variance, $\tau^{2}$ (Veroniki et al., 2015). Due to difficulties in interpretation and comparison of $\tau^{2}$ values across meta-analysis, an often used statistics to describe the extent of heterogeneity is the $I^{2}$ statistic (Higgins \& Thompson, 2002). $I^{2}$ expresses the percentage of the estimated variance that is due to variation in the true effect size. $I^{2}$ values of $0 \%$, $25 \%, 50 \%$ and $75 \%$ can be interpreted as no heterogeneity, small, medium and large heterogeneity, respectively (Higgins, Thompson, Deeks \& Altman 2003; Huedo-Medina, Sánchez-Meca, Marín-Martínez, \& Botella, 2006).

In the random effects meta-analysis the weights of each primary study, used to aggregate the results, are the inverse of the variance of $\operatorname{Var}\left(Y_{i}\right)$ in equation (2) where $\tau^{2}$ is replaced by its estimate. The estimated underlying grand mean in the random-effects model is the weighted average of the observed effect sizes (Cooper, Hedges \& Valentine, 2009; Borenstein et al., 2009).

\section{Example}

We generated an example dataset with known parameters, to illustrate the results of both the random effects meta-analysis, and later on, the results of the three BMAS methods. The true underlying effect size was a small Pearson correlation coefficient $r=.0997$, and $z(r)=0.1$ (Fisher transformed correlation), with medium heterogeneity $\left(I^{2}=50 \%\right)$, and $95 \%$ publication bias meaning that statistically non-significant had a probability of .05 to get published. We originally generated 15 studies, all having a sample size of 100. In this (arbitrary and fictitious) meta-analytic 
dataset, due to the publication bias, six studies ended up being published, and five out of six studies reached statistical significance.

The six effect sizes of the primary studies had Pearson correlations equal to 0.056 ( $p=$ $.291), 0.174(p=.042), 0.232(p=.010), 0.289(p=.002), 0.332(p=.0003)$, and $0.335(p=.0003)$.

Given the equal sample sizes, all studies have the same within study variance: $\frac{1}{N-3}=\frac{1}{97}=.0103$. Applying the random effects meta-analysis with the DerSimonian and Laird estimator (using the Metafor (Viechtbauer, 2010) package in R) resulted in summary estimate $z(r)=0.243,95 \%$ CI $[0.153,0.334], r=0.239,95 \%$ CI [0.152, 0.322], $\mathrm{p}<.0001$, indicating a small to medium effect size. The heterogeneity estimate equaled $\hat{\tau}^{2}=0.0025,95 \%$ CI $[0,0.0667]$, corresponding to $I^{2}=$ $19.41 \%, 95 \%$ CI $[0 \%, 86.6 \%]$. This heterogeneity estimate indicates small heterogeneity, but the 95\% confidence interval ranges from no to large heterogeneity, and the $Q$-test of homogeneity does not reach statistical significance $(Q(5)=6.204, p=.287)$.

\section{Bayesian Meta Analytic Snapshot (BMAS)}

BMAS can be considered as a method on the interface of frequentist and Bayesian statistics. It compares the likelihood of different models that could have generated the data; that is, different levels of the effect size and level of heterogeneity. The relative likelihood of these models is determined using Bayes' theorem and the user of the method can choose its own prior probabilities of the models.

A total of four effect sizes (no effect, small, medium and large effect) are considered, as well as four levels of heterogeneity (no heterogeneity, small, medium and large), resulting in 16 different models that are investigated for a meta-analytic dataset. The relative posterior likelihood indicates which of these models is most likely to be the underlying data generating model: Which effect size and heterogeneity are most compatible with the observed data. Different effect sizes metrics can be used, but for simplicity we will use correlations. With no effect, small, medium and large effect corresponding to $\rho=0, \rho=0.1, \rho=0.3$ and $\rho=0.5$, respectively (Cohen, 1988).

The four levels of heterogeneity correspond to $I^{2}=0 \%, 25 \%$ (small), 50\% (medium) and $75 \%$ (large). The value of $I^{2}$ depends on the so-called typical within-study variation, $\sigma^{2}$, and the between study variation, $\tau^{2}$. The typical within study variation is estimated using (Higgins \& Thompson, 2002, p. 1546):

$$
\hat{\sigma}^{2}=\frac{\sum w_{i}(k-1)}{\left(\sum w_{i}\right)^{2}-\sum w_{i}^{2}}
$$


where $k$ denotes the number of studies in the meta-analysis, and the weight of each study $w_{i}=\frac{1}{\sigma_{i}^{2}}$ is the weight of the fixed-effect meta-analysis model (a.k.a. equal-effect or common-effect model.. Since BMAS fixes the levels of $I^{2}$ to consider, it calculates the matching value of $\tau^{2}, \tau^{2}=\frac{I^{2} * \widehat{\sigma}^{2}}{1-I^{2}}$, for each of the four levels of $I^{2}$.

\section{Three variations of BMAS}

BMAS has three different variations. The first one, 'Uncorrected BMAS' assumes no publication bias, and is in that sense comparable to the random effects meta-analysis. The second method, (corrected) BMAS, does assume publication bias and aims to correct for it. It divides the effect sizes in two groups: statistically significant and non-significant effect sizes. BMAS assumes that all statistically significant effect sizes are equally likely to get published. All non-significant effect sizes are also assumed to have an equal probability to get published, but this probability may

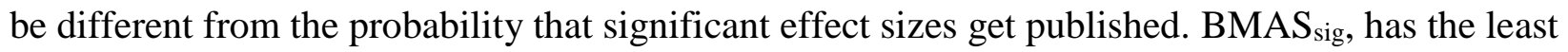
assumptions. It assumes publication bias, but only makes use of the statistically significant effect sizes and is, in that sense, comparable to methods such as the selection model by Hedges (1984), p-curve (Simonsohn et al., 2014) and p-uniform (van Assen et al., 2015). By only using the significant effect sizes BMAS $_{\text {sig }}$ does not have to assume anything about the publication probabilities of non-significant effect sizes. It does assume that all significant effect sizes have an equal probability to get published, as was also assumed in BMAS. Table 1 provides an overview of the BMAS methods.

Table 1. The likelihood equations of the three BMAS methods.

Method

Uncorrected BMAS

\section{Likelihood}

$$
g\left(\operatorname{data} \mid \mu, \tau^{2}\right)=\prod_{i=1}^{K} f\left(Y_{i} \mid \mu, \tau^{2}\right)
$$

Eq.

BMAS

$$
g\left(\operatorname{data} \mid \mu, \tau^{2}\right)=\prod_{i=1}^{K_{s}} \frac{f\left(Y_{i}^{\text {sig }} \mid, \tau^{2}\right)}{F\left(Y_{i}^{\text {sig }} \geq Y_{i}^{c v} \mid \mu, \tau^{2}\right)} \times \prod_{i=1}^{K_{n s}} \frac{f\left(Y_{i}^{n s} \mid \mu, \tau^{2}\right)}{F\left(Y_{i}^{n s}<Y_{i}^{c v} \mid \mu, \tau^{2}\right)}
$$


BMAS $_{\text {sig }}$

$$
g\left(\text { data } \mid \mu, \tau^{2}\right)=\prod_{i=1}^{K_{s}} \frac{f\left(Y_{i}^{s i g} \mid \mu, \tau^{2}\right)}{F\left(Y_{i}^{s i g} \geq Y_{i}^{c v} \mid \mu, \tau^{2}\right)}
$$

Note: $f$ refers to the normal density function, $F$ to the normal (de)cumulative distribution function, $g$ for the joint density function. $K$ refers to the total number of studies, whereas $i$ is used as index of the individual study results. $Y$ is the effect size of the individual study. The superscripts $n s$ (Eq. 4) and sig (eq. 4 and eq. 5) indicate the statistically non-significant and significant studies, respectively. The superscript $c v$ indicates the critical value of an individual effect size, which determines whether the effect size in a study is statistically significant or not.

\section{Uncorrected BMAS.}

Uncorrected BMAS evaluates the joint density of the data, $g\left(\right.$ data $\left.\mid \mu, \tau^{2}\right)$, using Equation (3), conditional on parameters $\mu$ and $\tau^{2}$. In order to obtain the posterior probability of each model, $\mathrm{P}\left(\mu_{a}, \tau^{2}{ }_{b} \mid\right.$ data $)$, we use Equation (6), to evaluate the likelihood of each model relatively to the fifteen other models.

$$
P\left(\mu_{a}, \tau_{b}^{2} \mid \text { data }\right)=\frac{g\left(\text { data } \mid \mu_{a}, \tau^{2}{ }_{b}\right)}{\sum_{i=1}^{4} \sum_{j=1}^{4} g\left(\operatorname{data} \mid \mu_{i}, \tau^{2}{ }_{j}\right)}
$$

Equation (6) follows directly from Bayes theorem after assuming a uniform prior, that is, that each of the sixteen models is equally likely underlying the meta-analytic data. Later on, we explain that it is straightforward to compute posterior probabilities using other priors, given the estimated posterior probabilities using a uniform prior with (6).

Higher posterior probabilities indicate a better match between the model and the observed data. Higher (lower) model probabilities than 1/16 indicate that this model has become more (less) likely after 'seeing' the data, than before (relative to the [average of] other models). Furthermore, differences in the posterior model probabilities express the precision. Larger differences in the 16 probabilities reflect higher precision.

In line with the Bayes Factor, we calculate the ratios of the likelihoods to express the relative likelihood of one model compared to the other models. The Bayes Factor expresses the "posterior odds of the null hypothesis when the prior probability on the null is one-half" (Kass \& Raftery, 1995, p. 773). The Bayes Factor is an often used statistic for model evaluation, but only compares two different models. Here, we compare one model with 15 other models, by taking the sum of these 15 other likelihoods. To avoid confusion, we will name this the BMAS Factor. Similar 
to the Bayes Factor, the BMAS Factor can only be derived from the posterior probabilities when a uniform prior has been used. For example, when a uniform prior is used, a posterior probability of .75 is equal to a BMAS Factor of 3 , which means that this particular model is three times more likely than all the fifteen other models together, after incorporating the data.

A Bayes Factor of 3 can be interpreted as positive evidence in favor of that model (Kass \& Raftery, 1995). The BMAS Factor can be interpreted in the same way. The BMAS Factor is smaller than the Bayes Factor and hence more conservative, since it compares one model with fifteen other models. As many models are very different (e.g., the null model with no effects versus the models where either average effect size or heterogeneity is large), the BMAS Factor may be - say - five times smaller than the Bayes Factor comparing the two best-fitting models in case of little data (e.g., two studies), and about two times or less smaller in case of a lot of data (e.g., ten studies or more). Thus, by using a benchmark of 3 for signaling positive evidence with the BMAS Factor, stronger evidence needs to be obtained than with a Bayes Factor of 3.

Finally, note that both Bayes Factors and BMAS Factors only tell us something about the probability of data, conditional on the models (Tendeiro \& Kiers, 2019). If none of our models is very probable, BMAS will provide most evidence in favor of the model(s) closest to 'the truth'.

\section{BMAS.}

BMAS adapts the previous method by correcting for publication bias. It does so by making a distinction between statistically significant and non-significant effect sizes, for a given $\alpha$ and the significance test being either one- or two-tailed (in all examples and the simulation study in this article we will use $\alpha=.05$ and one-tailed testing). The likelihoods of significant and non-significant effect sizes are calculated as conditional probability densities, conditional on being significant and non-significant, respectively, given values of true effect size and heterogeneity, using Equation (4), similar to $p$-uniform* (van Aert \& van Assen, 2021).

Intuitively, BMAS corrects for publication bias by taking into account the probability of a study being significant or non-significant (i.e., by dividing by this probability in the denominator of Equation 4). As publication bias affects this probability, publication bias is eliminated, differently from selection models that incorporate a parameter to estimate this probability of becoming significant or selected. In words, what BMAS does is evaluating the evidence on true effect size and heterogeneity after eliminating publication bias for the sets of significant and non- 
significant effect sizes separately, and then combine the evidence of both sets by multiplying their resulting likelihoods.

\section{BMAS sig.}

BMAS $_{\text {sig }}$ with the likelihood function in Equation (5) is like BMAS but does not take the non-significant effect sizes into account (i.e., it drops the likelihood of non-significant effect sizes from Equation (4)), similar to $p$-curve (Simonsohn et al., 2014) and p-uniform (van Assen et al., 2015). As such, it corrects for publication bias as BMAS but does not make the perhaps strong assumption that publication bias influences the publication probability of all non-significant effect sizes in the same way.

Note that if there are only statistically significant effect sizes available, BMAS and BMAS $_{\text {sig }}$ have the same likelihoods and will end up with the same results. By incorporating non-

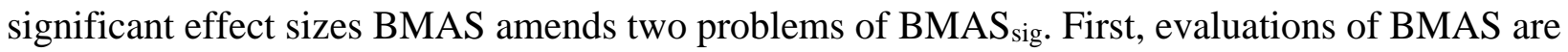
more efficient (i.e., more precise) than of $\mathrm{BMAS}_{\text {sig }}$ as it uses more information. This is particularly so if more non-significant effect sizes are present, when true effect size is small and publication bias minor. Second, in case of true effect size heterogeneity BMAS $_{\text {sig cannot well distinguish effect }}$ size from heterogeneity. That is, increasing the value of true effect size results in likelihoods that are similar to likelihoods that arise by increasing the value of true heterogeneity. Consequently, BMAS $_{\text {sig }}$ tends to over-evaluate the evidence of larger true effect sizes in case of effect size heterogeneity, which is similar to the problem of $p$-curve and $p$-uniform overestimating effect size in the case of heterogeneity (van Aert et al., 2016; van Aert, \& van Assen, 2021; van Assen et al., 2015).

Example

For illustration we apply the three variants of BMAS to the same example data with six effect sizes that we used to illustrate the random effects meta-analysis. Table 2 presents the posterior probabilities of all 16 models based on uncorrected BMAS, and of all four marginal posterior probabilities of true effect sizes and heterogeneity. These posterior probabilities are calculated using a uniform prior. Posterior probabilities larger than .0625 reflect an increase in evidence in favor of that model (relative to the 'average evidence' in favor of other models) after seeing the data, whereas marginal posterior probabilities larger than .25 reflect an increase of evidence in favor of the true effect size or heterogeneity. 
Table 2. Uncorrected BMAS: Posterior probabilities of each of the 16 models, using a uniform prior

\begin{tabular}{llllll}
\hline & $\rho=0$ & $\rho=0.1$ & $\rho=0.3$ & $\rho=0.5$ & \\
$I^{2}=0 \%$ & $2.96 \mathrm{e}-08$ & $2.32 \mathrm{e}-03$ & 0.244 & $1.26 \mathrm{e}-12$ & 0.246 \\
$I^{2}=25 \%$ & $2.00 \mathrm{e}-06$ & $9.38 \mathrm{e}-03$ & $\mathbf{0 . 3 0 8}$ & $1.05 \mathrm{e}-09$ & $\mathbf{0 . 3 1 8}$ \\
$I^{2}=50 \%$ & $9.51 \mathrm{e}-05$ & 0.026 & 0.273 & $6.20 \mathrm{e}-07$ & 0.300 \\
$I^{2}=75 \%$ & $1.91 \mathrm{e}-03$ & 0.032 & 0.102 & $1.54 \mathrm{e}-04$ & 0.136 \\
& 0.002 & 0.070 & $\mathbf{0 . 9 2 8}$ & $1.55 \mathrm{e}-04$ & \\
\hline
\end{tabular}

The results of the Uncorrected BMAS method are in line with the results of the random effects meta-analysis. The method indicates quite strong evidence in favor of a medium effect size $(\mathrm{P}(\rho=0.3)=.928$, BMAS Factor $=12.89)$, but provides imprecise evidence of heterogeneity with posterior probabilities that do not exceed $.318\left(\mathrm{P}\left(I^{2}=25 \%\right)=.318\right.$, BMAS Factor $\left.=0.46\right)$. Consequently, none of the 16 models got convincing support, with most (but weak) evidence in favor or the model with medium effect size and small heterogeneity $\left(\mathrm{P}\left(\rho=0.3, I^{2}=25 \%\right)=.308\right.$, BMAS Factor $=0.45)$.

Comparing the BMAS Factor to the Bayes Factor for illustrative purposes, the Bayes Factor of the best fitting model compared to the second best fitting model are (BMAS Factor between brackets) 13.26 (12.89) for average effect size marginalized over the different levels of heterogeneity, $1.06(0.46)$ for heterogeneity marginalized over the different levels of average effect size, and $1.13(0.45)$ for the joint model. Hence, the BMAS Factor is between 1 to 2.51 times smaller than the Bayes Factor in this example.

Table 3. BMAS: Posterior probabilities of each of the 16 models, using a uniform prior

\begin{tabular}{llllll}
\hline & $\rho=0$ & $\rho=0.1$ & $\rho=0.3$ & $\rho=0.5$ & \\
$I^{2}=0 \%$ & $1.80 \mathrm{e}-03$ & 0.052 & 0.084 & $2.73 \mathrm{e}-10$ & 0.137 \\
$I^{2}=25 \%$ & 0.014 & 0.127 & 0.090 & $3.44 \mathrm{e}-08$ & 0.231 \\
$I^{2}=50 \%$ & 0.071 & $\mathbf{0 . 2 0 8}$ & 0.074 & $2.95 \mathrm{e}-06$ & $\mathbf{0 . 3 5 3}$ \\
$I^{2}=75 \%$ & 0.119 & 0.130 & 0.030 & $1.08 \mathrm{e}-04$ & 0.279 \\
& 0.206 & $\mathbf{0 . 5 1 6}$ & 0.278 & $1.11 \mathrm{e}-04$ & \\
\hline
\end{tabular}


The second model, BMAS (Table 3), corrects for publication bias. This method provides most evidence for a small effect $(\mathrm{P}(\rho=0.1)=.516$, BMAS Factor $=1.07)$ and for medium heterogeneity $\left(\mathrm{P}\left(I^{2}=50 \%\right)=.353\right.$, BMAS Factor $\left.=0.55\right)$. Its combination is most probable $\left(\mathrm{P}\left(\rho=0.1 \& I^{2}=50 \%\right)=.208\right.$, BMAS Factor $\left.=0.26\right)$. The results however, express more uncertainty (i.e., less divergent posterior joint probabilities as well as marginal posterior probabilities) compared to the Uncorrected BMAS method. The fact that BMAS and uncorrected BMAS provide clearly different results may indicate that publication bias affected the meta-analysis.

Table 4. BMAS ${ }_{\text {sig: }}$ Posterior probabilities of each of the 16 models, using a uniform prior

\begin{tabular}{llllll}
\hline & $\rho=0$ & $\rho=0.1$ & $\rho=0.3$ & $\rho=0.5$ & \\
$I^{2}=0 \%$ & $1.63 \mathrm{e}-03$ & 0.034 & 0.124 & $2.48 \mathrm{e}-09$ & 0.160 \\
$I^{2}=25 \%$ & 0.014 & 0.092 & 0.099 & $1.26 \mathrm{e}-07$ & 0.204 \\
$I^{2}=50 \%$ & 0.078 & $\mathbf{0 . 1 7 1}$ & 0.065 & $4.82 \mathrm{e}-06$ & 0.313 \\
$I^{2}=75 \%$ & 0.160 & 0.137 & 0.026 & $1.01 \mathrm{e}-04$ & $\mathbf{0 . 3 2 3}$ \\
& 0.253 & $\mathbf{0 . 4 3 3}$ & 0.313 & $1.06 \mathrm{e}-04$ & \\
\hline
\end{tabular}

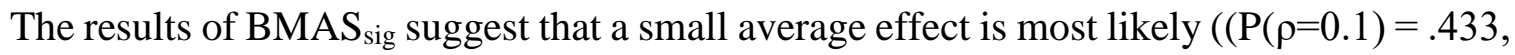
BMAS Factor $=0.76))$, whereas large heterogeneity $\left(\mathrm{P}\left(I^{2}=75 \%\right)=.323\right.$, BMAS Factor $\left.=0.48\right)$ and medium heterogeneity $\left(\mathrm{P}\left(I^{2}=50 \%\right)=.313\right.$, BMAS Factor $\left.=0.46\right)$ are about equally likely (Table 4). Agreeing with BMAS, the model with small effect size and medium heterogeneity gets most support $\left(\mathrm{P}\left(\rho=0.1 \& I^{2}=50 \%\right)=.171\right.$, BMAS Factor $\left.=0.21\right)$. Note that evidence in favor of any model is weak, which was also the case for uncorrected BMAS and BMAS. Five studies with a sample size of 100 are often simply not enough to distinguish models with different effect sizes and particularly different heterogeneity.

\section{Prior probabilities}

The results presented in Tables 2-4 are based on uniform priors. Consequently, the posterior probabilities solely reflect model differences in the (conditional) likelihood. However, a uniform prior may be neither desirable nor realistic. For instance, on the basis of previous empirical evidence researchers may expect a zero true effect size and no effect size heterogeneity in a large replication project (e.g., based on the many labs projects, Klein et al., 2014; Olsson-Collentine, Wicherts, \& van Assen, 2020). Alternatively, a meta-analyst may have used very broad inclusion 
criteria for the studies, with a wide range of populations, treatments, and measurements of both independent and dependent variables as is mainstream in meta-analyses in psychology, and therefore expecting large heterogeneity of effect size (van Erp, Verhagen, Grasman, \& Wagenmakers, 2017). Or one may use standard priors for both effect size and heterogeneity to derive the prior probabilities of the models.

With BMAS it is computationally straightforward to re-compute the posterior probabilities based on another prior without having to re-do the meta-analysis. The re-computed posterior probabilities $P\left(\mu, \tau^{2} \mid \text { data }\right)^{*}$ can be computed using

$$
P\left(\mu, \tau^{2} \mid \text { data }\right)^{*}=\frac{P\left(\mu, \tau^{2} \mid \text { data }\right) * P\left(\mu, \tau^{2}\right)}{\sum_{\mu=1}^{4} \sum_{\tau^{2}=1}^{4} P\left(\mu, \tau^{2} \mid \text { data }\right) * P\left(\mu, \tau^{2}\right)}
$$

with $P\left(\mu, \tau^{2} \mid\right.$ data $)$ and $\mathrm{P}\left(\mu, \tau^{2}\right)$ being the posterior probability based on the uniform prior and the updated alternative prior, respectively.

Our paper concludes with a shiny-app that allows the researcher to specify the alternative prior. The shiny-app also includes two standard alternative priors. First, the conservative uniform prior with parameter $\pi_{0}>1$ puts weight $\pi_{0}$ on the model with a zero true effect size and no heterogeneity, assuming the effect is always zero, and $1 /\left(15+\pi_{0}\right)$ on each of the other models. Note that for $\pi_{0}=1$ we obtain the default uniform prior. For example, if a researcher believes that the 'no effect model' is just as likely as all the other models together (i.e., $\pi_{0}=15$ ), then the posterior probability of the no effect model under the BMAS model increases from $\operatorname{Pr}\left(\rho=0 \& I^{2}=0 \%\right)=$ 0.0018 (see upper left cell of Table 3) to $\operatorname{Pr}\left(\rho=0 \& I^{2}=0 \%\right)=0.026$. Although this increase is substantial, the support for the null model remains negligible.

The second standard prior is based on statistically independent normal distributions for both the marginal prior on the effect size and the heterogeneity. Here, the prior on the (Fischer transformed) correlation is a normal distribution with as default parameters a mean of 0 and a variance of 1 . This is a relatively non-informative prior, due to its large variance.

For heterogeneity, many priors have been suggested in the literature, such as the inversegamma distribution, the half-Cauchy distribution, or the half-normal distribution. Some of these priors are based on empirical data (e.g. van Erp et al., 2017), while other are chosen because of their theoretical properties (Williams, Rast \& Bürkner, 2018). The use of a uniform prior can be a good starting point, but puts relatively much weight on unreasonably large values (Gelman, 2006; Williams et al., 2018). As an alternative to a uniform prior, one could use a half-normal distribution 
centered at 0 , with a large standard deviation (Gelman, 2006). The inverse gamma distribution, as advised by van Erp et al. (2017) can be problematic especially in the context of meta-analysis, since its results depend strongly on the scale and shape parameter $(\alpha$ and $\beta)$ in the boundary of the sample space (when $\tau^{2}$ is close to 0) (Gelman, 2006; Williams et al., 2018). While half- $t$ distributions, such as the half-Cauchy distribution, are more flexible and behave better near 0 , a simulation study by Williams et al. (2018) showed that both the inverse-gamma prior and the halfCauchy prior only provided non-zero estimates. This property is not desirable for the estimation of heterogeneity, where $\tau^{2}=0$ can be a perfectly acceptable estimate. Therefore, we use a halfnormal distribution $\tau^{2} \sim \mathbb{N}\left(\mu, \sigma^{2}\right)$ with only positive values as marginal prior of $\tau^{2}$.

Importantly, note that the prior is on $\tau^{2}$ rather than on $I^{2}$, which implies that when transforming the prior on $\tau^{2}$ to $I^{2}, I^{2}$ is affected by both the prior on $\tau^{2}$ and the within-study variances. That is, for the same prior on $\tau^{2}$, more imprecise studies with a large variance (i.e., studies with a small sample size) yield higher prior probabilities on small $I^{2}$, whereas large study samples yield higher prior probabilities on large $I^{2}$. Note that the mode of our default prior indicates homogeneity $\left(\tau^{2}=0\right)$. We argue this is a sensible choice as we see within replication that when the true effect size is 0 , there is often also no heterogeneity (Olsson-Collentine, et al., 2020). Given our default prior of $\rho \sim \mathbb{N}(0,1)$, choosing no heterogeneity is a logical prior on heterogeneity. As default values for our prior we use $\tau^{2} \sim \mathbb{N}(0,0.5)$, implying a rather non-informative prior of effect size heterogeneity. Consequently, it will not have much impact on the posterior probabilities of the model. Of course, this can be changed if a researcher has reason to use a more informative prior.

Table 5 shows the posterior model probabilities of BMAS applied to our examples with six studies, based on the default priors of effect size and heterogeneity. The posterior probabilities are obtained by multiplying the marginal priors on effect size and the implied marginal prior on $I^{2}$ to obtain the joint prior, and updated the posterior probabilities using Equation (7).

Table 5. BMAS: Posterior probabilities of each of the 16 models with priors $\rho \sim \mathbb{N}(0,1), \tau^{2} \sim$ $\mathbb{N}(0,0.5)$

\begin{tabular}{llllll}
\hline & $\rho=0$ & $\rho=0.1$ & $\rho=0.3$ & $\rho=0.5$ & \\
$I^{2}=0 \%$ & $1.80 \mathrm{e}-03$ & 0.052 & 0.081 & 0 & 0.135 \\
$I^{2}=25 \%$ & 0.015 & 0.128 & 0.088 & 0 & 0.230 \\
\hline
\end{tabular}




\begin{tabular}{llllll}
\hline$I^{2}=50 \%$ & 0.072 & $\mathbf{0 . 2 1 0}$ & 0.072 & 0 & $\mathbf{0 . 3 5 4}$ \\
$I^{2}=75 \%$ & 0.120 & 0.131 & 0.030 & $1 \mathrm{e}-04$ & 0.281 \\
& 0.209 & $\mathbf{0 . 5 2 1}$ & 0.270 & $1 \mathrm{e}-04$ & \\
\hline
\end{tabular}

If we compare these results to the results with a uniform prior, we only obtain small differences (e.g., the posterior probability of the best fitting model is .210, whereas it was .208 using a uniform prior). Of course, using larger values of $\mu$ and/or smaller values of $\sigma^{2}$ in our prior, will result in a bigger impact of the priors on the posterior probabilities.

\section{The performance of the BMAS methods: a simulation study}

To assess the performance of each of the three BMAS methods, a Monte Carlo simulation study was conducted. A fully crossed design was used for the 4 (effect size) $\times 4$ (effect size heterogeneity) $\times 4$ (publication bias) $\times 2$ (sample size) $\times 3$ (number of published studies) design, resulting in 384 different conditions. In each of these conditions, 10,000 meta-analytic datasets were generated that were analyzed by uncorrected BMAS and BMAS. Some of these datasets however, did not contain any statistically significant studies (e.g., in the datasets with no publication bias and true effect size equal to zero). To make sure that all three methods had the same number of iterations, data generation for $\mathrm{BMAS}_{\text {sig }}$ continued until 10,000 datasets were available that contained at least one statistically significant effect size, whereas data generation for uncorrected BMAS and BMAS always ended after 10,000 datasets. For example, about 15,600 iterations were needed to obtain the 10,000 datasets in the condition in the conditions with $K=20$ without publication bias, and zero true effect size and no effect size heterogeneity. For other conditions less iterations were required as the probability of at least one statistically effect size in a set of studies increases with true effect size, heterogeneity, publication bias, the number of studies $(K)$, and the sample size $(N)$ in case of a nonzero effect.

Furthermore, an additional simulation study was run to assess the consistency of the evaluation of the methods. Consistency refers to the results converging to the truth when the number of studies in the meta-analysis is very large, that is, that the evaluation of effect size or heterogeneity corresponds to that of the true value, or in other words that the posterior probability of the correct model approaches 1 . A requirement of a good meta-analytic method is that its results are consistent under conditions of publication bias. In the simulation study on consistency, we used a fixed sample size $(\mathrm{N}=62)$ and a fixed very large number of published studies $(K=1,000)$. This 
simulation had 64 conditions $(4$ (effect size) $\times 4$ (heterogeneity) $\times 4$ (publication bias) $)$.

For the simulation study examining consistency $(K=1,000)$, all three versions of the BMAS method were applied to each meta-analytic dataset. For the other conditions $(K=5,20,50$ or 100$)$, we applied both random-effects meta-analysis as well as the three BMAS versions to each dataset. We evaluated the methods on their estimates of effect size and heterogeneity in case of randomeffects meta-analysis (DerSimonian and Laird method for estimating heterogeneity) and the posterior probabilities of the BMAS methods based on uniform priors, with $\alpha=.05$ and one-tailed testing.

All simulations were conducted in $\mathrm{C}++$, all code can be found in the supplemental materials on OSF (https://osf.io/98drq/).

\section{Data generation}

Primary study effect sizes were drawn from a normal distribution with a mean equal to the underlying true effect size, using Fisher transformed correlation, based on a Pearson correlation of $\rho=0$ (no effect), $\rho=0.1$ (small effect), $\rho=0.3$ (medium effect), and $\rho=0.5$ (large effect). And a

standard deviation equal to $\sqrt{\sigma^{2}+\tau^{2}}$, where $\sigma^{2}=\frac{1}{N-3}$ (of the Fisher-transformed correlation) and $\tau^{2}=\frac{I^{2} \widehat{\sigma}^{2}}{1-I^{2}}\left(\right.$ based on Higgins and Thompson, 2002). Heterogeneity varied from zero $\left(\mathrm{I}^{2}=0 \%\right)$, small $\left(\mathrm{I}^{2}=25 \%\right)$, medium $\left(\mathrm{I}^{2}=50 \%\right)$ to large $\left(\mathrm{I}^{2}=75 \%\right)$. Sample size, $N$, was either 62 (the median sample size in psychology studies, Hartgerink, Wicherts, \& van Assen, 2017) or 100. These sample sizes only varied between conditions, within a condition the study sample sizes were equal.

Each generated study was subjected to publication bias: statistically significant effect sizes ( $p<.05$, one-tailed) were assumed to be published with a probability of $100 \%$, whereas the probability of non-significant effect sizes $(p>.05)$ being published depended on publication bias. Publication bias varied from $0 \%$ bias (all non-significant effect sizes published), 50\% bias, 95\% bias, and 100\% bias (no non-significant effect sizes published). For the $50 \%$ and $95 \%$ bias conditions, non-significant studies had a 50\% and 5\% chance respectively, of 'getting published'. A meta-analytical dataset was complete when there were $K$ published (non-significant and/or significant) studies within a dataset, with $K$ being either 5, 20, 50 or 100 studies.

\section{Method evaluation}

For random effects meta-analysis we were interested in the average bias in the estimate of the effect size and heterogeneity, that is, the difference between the true values and the estimated 
values of Pearson's $r$ and $\tau^{2}$. For the three different BMAS methods, we were interested in six criteria; proportion of meta-analyses assigning the highest posterior probability to the (i) correct effect size, (ii) correct amount of heterogeneity, and (ii) correct model (both correct effect size and correct heterogeneity), and proportion of meta-analyses assigning a posterior probability of at least $75 \%$ (implying a Bayes Factor $>3$, when using uniform priors) to the (i) correct effect size, (ii) correct heterogeneity, and (iii) correct model. The last three criteria indicate that the method did not only point to the correct model, but also expresses certainty in its identification.

In the next section, we will subsequently discuss the consistency of the methods (when $\mathrm{K}=1,000$ ), the results for meta-analytical data-sets containing 20 studies and 100 studies. Finally, we will shortly discuss the results regarding extremely small meta-analysis $(K=5)$, since such small meta-analysis are very common in some fields, for instance medicine (Davey, Turner, Clarke \& Higgins, 2011). Since the results of moderate sized meta analysis $(K=50)$ are very much in line with what one would expect after seeing the $K=20$ and $K=100$ conditions, and since the impact of a larger sample size $(\mathrm{N}=100)$ is relatively small, results of these condition can be found in the supplemental materials to keep this article concise (https://osf.io/98drq/).

\section{Results}

\section{Consistency}

Figure 1 shows the proportion of correct inferences (both effect size and heterogeneity) for each of the three BMAS methods. This figure consists of 8 subplots. The left column of plots shows the proportion of correct inferences based on selecting the model with the highest posterior probability. The plots in the right column show the proportion of correct model inferences where the posterior probability exceeded $75 \%$, corresponding to a BMAS factor larger than 3 , which provides a more strict measure of performance than the previous criterion of performance. Publication bias increases from the first row (0\%) to the last row (100\%). Within each subplot, the

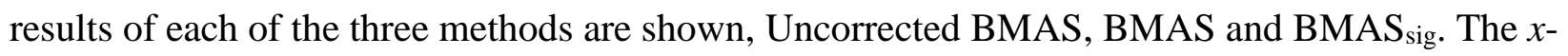
axis shows the different conditions, underlying true effect size and heterogeneity, whereas the $y$ axis provides the proportion of correct inferences. Ideally this proportion equals 1 . 
proportion correct

proportion correct

model

model $>75 \%$

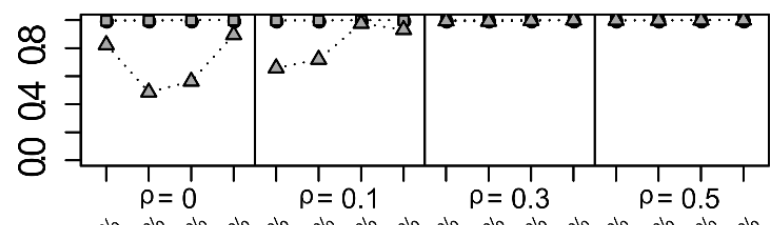

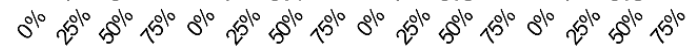
heterogeneity $\left(1^{2}\right)$

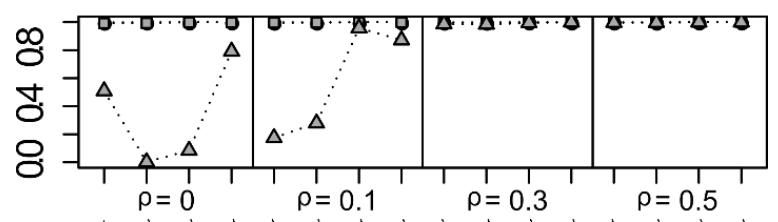

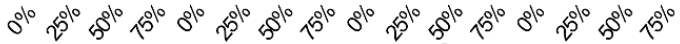
heterogeneity $\left({ }^{2}\right)$

$50 \%$ publication bias

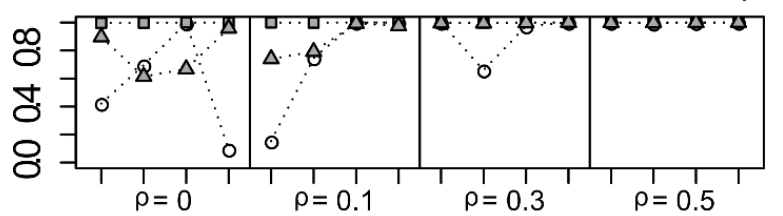

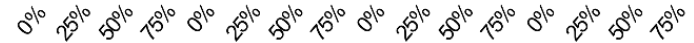
heterogeneity $\left(1^{2}\right)$

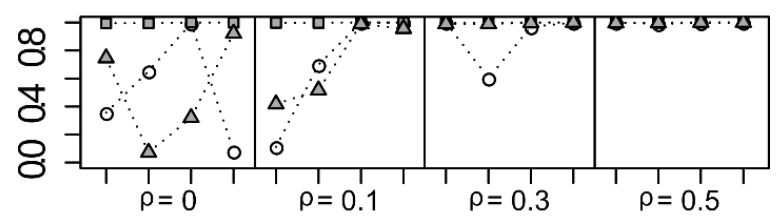

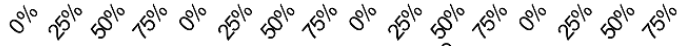
heterogeneity $\left({ }^{2}\right)$

95\% publication bias

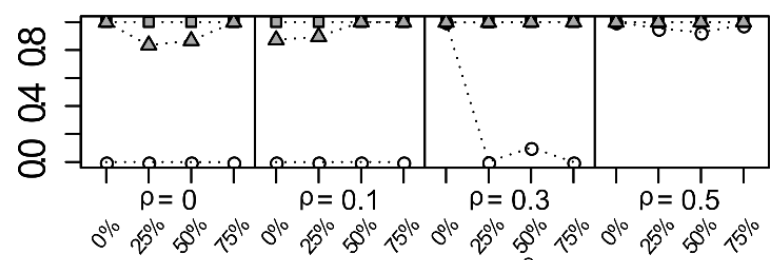

heterogeneity $\left(\left(^{2}\right)\right.$

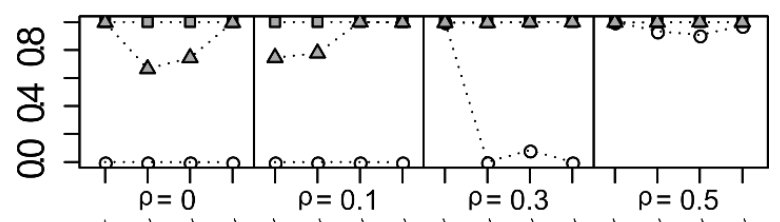

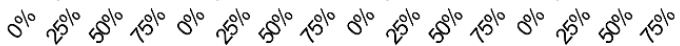
heterogeneity $\left(I^{2}\right)$

$100 \%$ publication bias

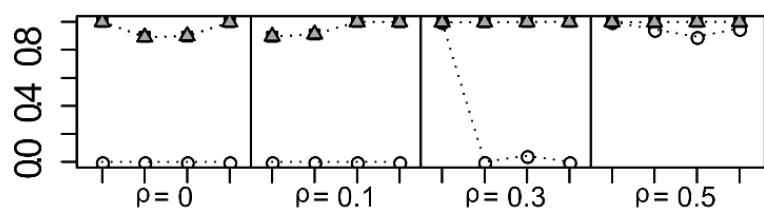

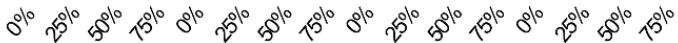
heterogeneity $\left(\mathrm{I}^{2}\right)$

o Uncorrected BMAS

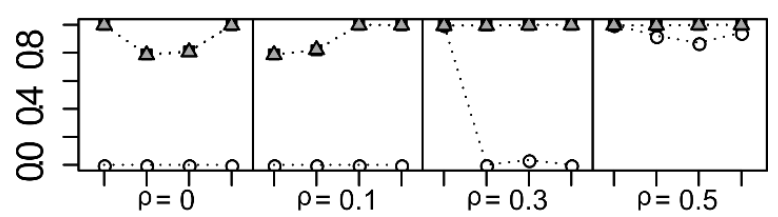

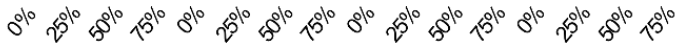
heterogeneity $\left({ }^{2}\right)$

$\square$ BMAS

$\triangle$ BMAS $_{\text {sig }}$

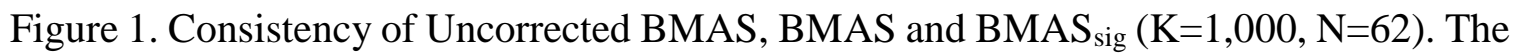
$y$-axis shows the proportion of correct effect size and heterogeneity evaluations (left) and correct evaluations with a posterior probability of at least $75 \%$ (right), based on a uniform prior, for different levels of publication bias (plot rows) and different levels of the true effect size and levels of heterogeneity ( $x$-axis). 
Posterior probabilities of uncorrected BMAS (white dots) were consistent when publication bias was absent (top row); it assigned $>75 \%$ probability to the correct model $99.9 \%$ of the times, in all conditions without publication bias. For larger values of publication bias $\left(2^{\text {nd }}, 3^{\text {rd }}\right.$ and $4^{\text {th }}$ row), the performance of uncorrected BMAS dropped substantially. When effect sizes were zero or small, or when there was severe publication bias (>95\% bias), the evaluation of uncorrected BMAS was not consistent but assigned a high posterior probability to the incorrect effect size and heterogeneity. Only when true effect size was large such that almost all studies reached statistical significance and publication bias had little impact, the performance of uncorrected BMAS was also good.

The posterior probabilities of BMAS (squares) were consistent. When there was no publication bias, it assigned $>75 \%$ probability to the correct model in $99.8 \%$ of all iterations. As publication bias increased, the performance of BMAS was hardly effected. Using the most strict criteria (i.e., having both the effect size and heterogeneity correct and doing so by appointing at least $75 \%$ certainty to it) BMAS was accurate in at least $99 \%$ of the iterations, in all but four conditions. When there was full publication bias and $\rho=0, I^{2}=25$ or $50 \%$ or if $\rho=0.1$, and $I^{2}=0$ or $25 \%$ the proportion of cases with posterior probabilities of at least .75 for the correct model was equal to $78.9,81.1,78.9$ and $81.9 \%$ respectively. The reason is that BMAS suffered from a lack of studies that were non-significant in these conditions with full publication bias. Increasing the number of studies in the meta-analysis to 10,000 in these conditions (results not shown) did result in accurate decisions in at least $99.99 \%$ of the iterations, demonstrating that BMAS is indeed consistent in these conditions.

The posterior probabilities of $\mathrm{BMAS}_{\mathrm{sig}}$ (triangle) were also consistent, but sometimes needed a lot more data than needed by BMAS. Particularly when effect sizes were small (left side of each plot) and there was little publication bias (top rows), BMAS $\mathrm{S}_{\text {sig }}$ had less data available, resulting in a relatively low performance and less certainty in its model choice. This can also be seen in the drop in performance when we require $75 \%$ posterior probabilities (right column). When sufficient data was available for this method as well $\left(\mathrm{K}=100,000\right.$, average $\mathrm{K}_{\text {sig }}$ in all conditions $\geq$ 5000 , results not shown), $\mathrm{BMAS}_{\text {sig }}$ also resulted in accurate decisions with $>75 \%$ certainty in at least $99.95 \%$ of the iterations, demonstrating that $\mathrm{BMAS}_{\text {sig }}$ is also consistent.

To conclude, Uncorrected BMAS is generally inconsistent and its performance dropped in the presence of publication bias. BMAS $\mathrm{sig}_{\mathrm{sig}}$ and BMAS are both consistent, also when publication 
bias is present, demonstrating that the methods adequately adjusted for publication bias in the

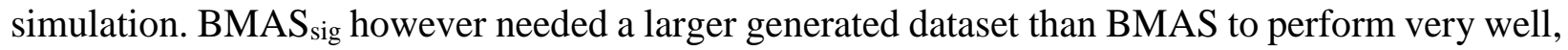
since it only uses the statistically significant studies.

\section{Meta-analysis on 20 studies}

The results of random effects meta-analysis are shown in Table 6. The left columns depict average bias in the effect size estimate $(\hat{\rho}-\rho)$, using Pearson's $r$, for different values of the true effect sizes (in columns) and heterogeneity (in rows). Positive values indicate that the effect size was overestimated on average, while negative values imply an underestimation. The four columns to the right depict findings on bias in the estimates of heterogeneity $\left(\widehat{I}^{2}-I^{2}\right)$. Each block of four rows provide the results for different levels of publication bias.

Table 6. Average bias in effect size estimate $(\rho)$ and heterogeneity $\left(\mathrm{I}^{2}\right)$ of random effects metaanalysis, $K=20, N=62$ (standard deviations of meta-analytic estimates between brackets)

\begin{tabular}{|c|c|c|c|c|c|c|c|c|}
\hline \multirow{7}{*}{$\begin{array}{l}I^{2}=0 \% \\
I^{2}=25 \% \\
I^{2}=50 \% \\
I^{2}=75 \%\end{array}$} & \multicolumn{4}{|c|}{ Bias in $\rho$} & \multicolumn{4}{|c|}{ Bias in $\mathrm{I}^{2}$} \\
\hline & $\rho=0$ & $\rho=0.1$ & $\rho=0.3$ & $\rho=0.5$ & $\rho=0$ & $\rho=0.1$ & $\rho=0.3$ & $\rho=0.5$ \\
\hline & \multicolumn{8}{|c|}{ 0\% publication bias } \\
\hline & $0.00(0.03)$ & $0.00(0.03)$ & $0.00(0.03)$ & $0.00(0.02)$ & $0.06(0.11)$ & $0.06(0.10)$ & $0.06(0.11)$ & $0.06(0.11)$ \\
\hline & $0.00(0.03)$ & $0.00(0.03)$ & $0.00(0.03)$ & $0.00(0.02)$ & $-0.08(0.17)$ & $-0.08(0.17)$ & $-0.08(0.17)$ & $-0.08(0.17)$ \\
\hline & $0.00(0.04)$ & $0.00(0.04)$ & $0.00(0.04)$ & $0.00(0.03)$ & $-0.11(0.19)$ & $-0.11(0.19)$ & $-0.11(0.19)$ & $-0.11(0.19)$ \\
\hline & $0.00(0.06)$ & $0.00(0.06)$ & $0.00(0.05)$ & $0.00(0.04)$ & $-0.06(0.11)$ & $-0.06(0.11)$ & $-0.06(0.11)$ & $-0.06(0.11)$ \\
\hline & \multicolumn{8}{|c|}{$\mathbf{5 0 \%}$ publication bias } \\
\hline$I^{2}=0 \%$ & $0.01(0.03)$ & $0.03(0.03)$ & $0.02(0.02)$ & $0.00(0.02)$ & $0.10(0.14)$ & $0.10(0.13)$ & $0.03(0.07)$ & $0.05(0.10)$ \\
\hline $\mathrm{I}^{2}=25 \%$ & $0.02(0.04)$ & $0.04(0.03)$ & $0.03(0.03)$ & $0.00(0.02)$ & $0.01(0.18)$ & $-0.02(0.18)$ & $-0.15(0.14)$ & $-0.10(0.16)$ \\
\hline$I^{2}=50 \%$ & $0.03(0.04)$ & $0.05(0.04)$ & $0.03(0.03)$ & $0.00(0.03)$ & $-0.01(0.16)$ & $-0.05(0.18)$ & $-0.20(0.20)$ & $-0.14(0.19)$ \\
\hline \multirow[t]{2}{*}{$\mathrm{I}^{2}=75 \%$} & $0.06(0.06)$ & $0.07(0.06)$ & $0.05(0.05)$ & $0.02(0.04)$ & $-0.02(0.09)$ & $-0.05(0.11)$ & $-0.11(0.14)$ & $-0.10(0.13)$ \\
\hline & \multicolumn{8}{|c|}{$95 \%$ publication bias } \\
\hline$I^{2}=0 \%$ & $0.13(0.04)$ & $0.14(0.02)$ & $0.04(0.02)$ & $0.00(0.02)$ & $0.30(0.17)$ & $0.02(0.07)$ & $0.00(0.02)$ & $0.05(0.09)$ \\
\hline $25 \%$ & $0.17(0.04)$ & $0.16(0.03)$ & $0.06(0.02)$ & $0.00(0.02)$ & $0.09(0.19)$ & $-0.20(0.12)$ & $-0.24(0.04)$ & $-0.12(0.15)$ \\
\hline $\mathrm{I}^{2}=50 \%$ & $0.21(0.04)$ & $0.18(0.03)$ & $0.08(0.03)$ & $0.01(0.03)$ & $-0.12(0.2$ & $-0.37(0.18)$ & $-0.41(0.14)$ & $-0.19(0.18)$ \\
\hline \multirow[t]{2}{*}{$\mathrm{I}^{2}=75 \%$} & $0.28(0.04)$ & $0.23(0.04)$ & $0.12(0.03)$ & $0.03(0.04)$ & $-0.25(0.25)$ & $-0.38(0.25)$ & $-0.36(0.21)$ & $-0.17(0.14)$ \\
\hline & \multicolumn{8}{|c|}{$100 \%$ publication bias } \\
\hline $\mathrm{I}^{2}=0 \%$ & $0.26(0.01)$ & $0.18(0.01)$ & $0.05(0.02)$ & $0.00(0.02)$ & $0.00(0.00)$ & $0.00(0.00)$ & $0.00(0.01)$ & $0.04(0.09)$ \\
\hline $\mathrm{I}^{2}=25 \%$ & $0.27(0.01)$ & $0.19(0.01)$ & $0.06(0.02)$ & $0.00(0.02)$ & $-0.25(0.00)$ & $-0.25(0.00)$ & $-0.24(0.04)$ & $-0.12(0.15)$ \\
\hline $\mathrm{I}^{2}=50 \%$ & $0.30(0.02)$ & $0.22(0.02)$ & $0.08(0.02)$ & $0.01(0.03)$ & $-0.50(0.01)$ & $-0.50(0.02)$ & $-0.44(0.11)$ & $-0.19(0.18)$ \\
\hline$I^{2}=75 \%$ & $0.35(0.02)$ & $0.27(0.03)$ & $0.13(0.03)$ & $0.04(0.04)$ & $-0.70(0.11)$ & $-0.64(0.16)$ & $-0.42(0.20)$ & $-0.18(0.14)$ \\
\hline
\end{tabular}

Starting with the results on effect size, the effect size was estimated correctly in case of no publication bias. Confirming to the literature (e.g., Borenstein, et al., 2009; Nuijten, et al., 2015; van Assen et al., 2015), we saw on average overestimation that increased in publication bias and heterogeneity, but decreased in true effect size. Concerning heterogeneity, random effects meta- 
analysis with the DerSimonian and Laird estimator yielded biased estimates in almost all conditions, even if there was no publication bias. $I^{2}$ was overestimated in case of homogeneity, as estimates of $I^{2}$ cannot be lower than 0 . Striking was the strong underestimation of heterogeneity in case of strong publication bias (95\% or $100 \%)$, where $I^{2}$ was very often estimated equal to 0 , even when true heterogeneity was medium. However, we should be reluctant to interpret and generalize these results, as the effect of publication bias on the estimates of heterogeneity is complex and non-linear (Augusteijn et al., 2019). For instance, Augusteijn et al. showed that the strong underestimation here is a consequence of all studies having the same sample size, and that in these conditions one can also obtain overestimation of heterogeneity if studies strongly differ in sample size. The complex and non-linear relation between the estimated heterogeneity and the underlying true effect size, true heterogeneity, the sample size and level of publication bias makes general claims impossible, as gross underestimation can easily transform into overestimation.

\section{Performance BMAS: average effect size}

Figure 2 shows the proportion of correct effect size evaluations, and has the same structure as Figure 1. Twenty studies were hardly ever enough to determine the correct effect size with certainty (>75\%, right column); the methods did seldom yield conclusive evidence on the right model. Only when the effect size is large, in combination with large heterogeneity, there was sufficient evidence in the effect size choice for all BMAS methods. This was due to this model being further apart from the other models (i.e., the difference between an effect size of $r=.3$ and $r$ $=.5$ is relatively large, and there is no model with a higher value than $r=.5$ ). 
proportion correct effect size

$0 \%$ publication bias

proportion correct

effect size $>75 \%$

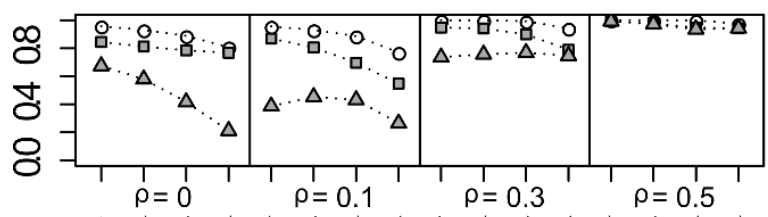

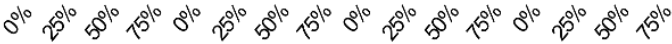
heterogeneity $\left(I^{2}\right)$

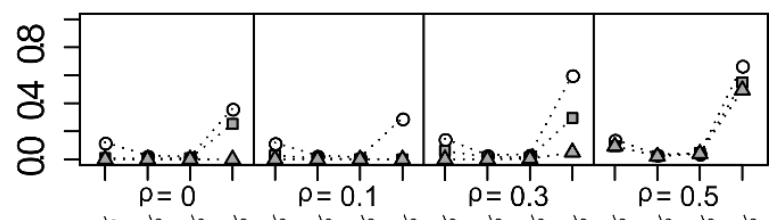

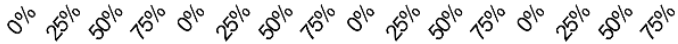
heterogeneity $\left(I^{2}\right)$

$50 \%$ publication bias

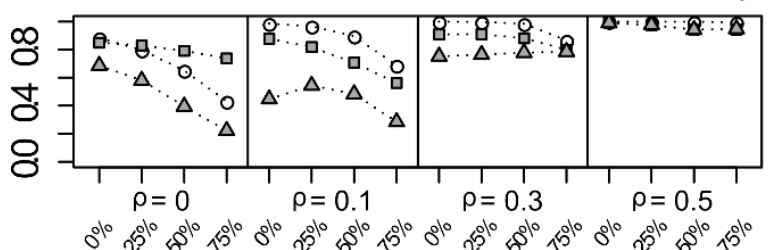

की heterogeneity $\left(1^{2}\right)$

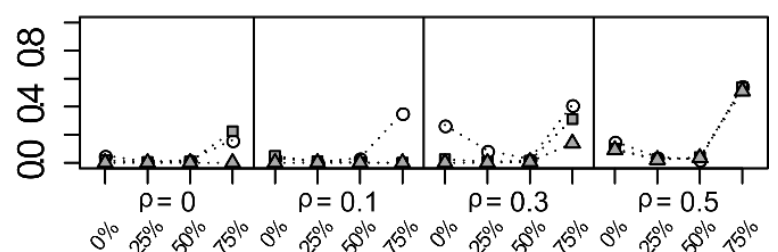

heterogeneity $\left(\mathrm{I}^{2}\right)$

95\% publication bias

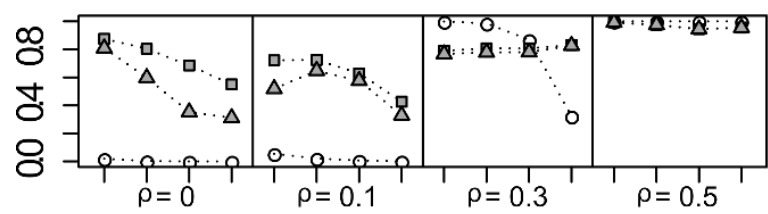

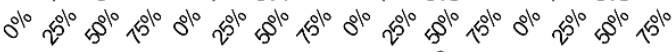
heterogeneity $\left(\mathrm{I}^{2}\right)$

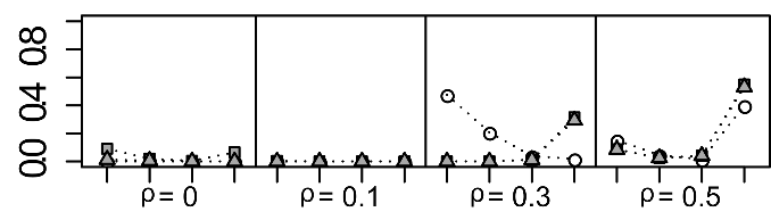

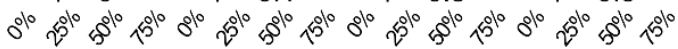
heterogeneity $\left(I^{2}\right)$

$100 \%$ publication bias

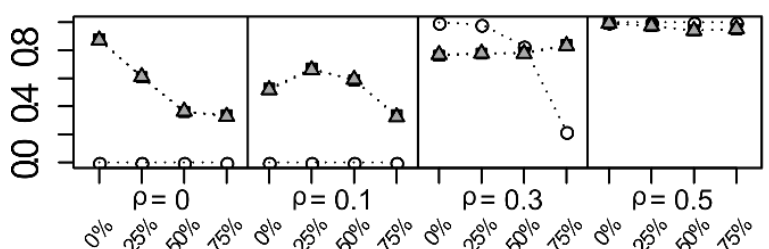

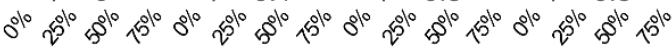
heterogeneity $\left(\mathrm{I}^{2}\right)$

o Uncorrected BMAS

口 BMAS

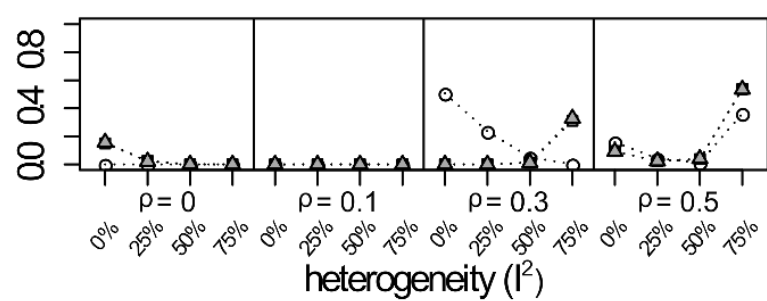

$\triangle$ BMAS $_{\text {sig }}$

Figure 2. Correct effect size evaluation of Uncorrected BMAS, BMAS and BMAS ${ }_{\text {sig }}(K=20$, $\mathrm{N}=62$ ). The $y$-axis shows the proportion of correct effect size evaluations (left) and correct evaluations with a posterior probability of at least $75 \%$ (right), based on a uniform prior, for publication bias (rows) and true effect size and heterogeneity ( $x$-axis).

When the true effect size was zero, small or medium, the performance differed per condition and per method. Uncorrected BMAS performed best if publication bias was absent (top left plot). When publication bias increased, the performance of Uncorrected BMAS, in terms of effect size evaluation, decreased, especially when the true effect size was absent or small. This is similar to the results of random effect meta-analysis. When there was full publication bias and the 
effect size was 0 or small, Uncorrected BMAS never chose the correct effect size model. Furthermore, the performance on average effect size evaluation of Uncorrected BMAS decreased when heterogeneity increased. For example, in case of $95 \%$ publication bias in combination with a medium true effect, the performance of Uncorrected BMAS dropped from $99.9 \%$ correct (no heterogeneity) to $32.2 \%$ correct (large heterogeneity).

BMAS performed similar to BMAS $\mathrm{Big}_{\mathrm{si}}$, or better. Especially when there was a relatively large number of non-significant studies (less bias, in combination with a null or small true effect)

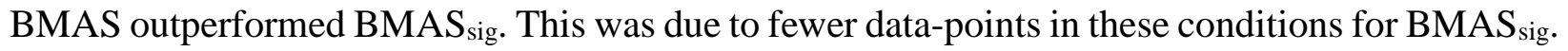
When publication bias increased, the performance of BMAS stayed relatively stable, and in the conditions that are found to be most realistic for psychology (that is, a small effect and large publication bias), BMAS performed best in evaluating the effect size.

\section{Performance BMAS: effect size heterogeneity}

Figure 3 shows the results of the three methods in terms of correct heterogeneity evaluation. Similar to the results on average effect size, strong evidence on the right model (>75\%) was rather rare. For example, averaged across all 64 conditions, BMAS evaluated $I^{2}$ correctly in $57.0 \%$ of all iterations, but found strong evidence for the correct heterogeneity estimate in only $7.9 \%$ of all iterations. Here too, performance was best when the 'extreme' model was true (i.e., no heterogeneity or large heterogeneity), as everything smaller than $I^{2}=0 \%$ (extreme homogeneity) and larger than $I^{2}=75 \%$, accumulate in these 'boundary' values.

Uncorrected BMAS performed worst with respect to choosing the correct heterogeneity, in most conditions when there was substantial or full publication bias (95\% or 100\%, lower left

column). The same pattern can also be observed in the $I^{2}$ estimates of random effects meta-analysis (see Table 6), and reflects the impact of publication bias on estimates of heterogeneity (Augusteijn et al., 2019). Performance of Uncorrected BMAS and BMAS was comparable when publication bias equaled $0 \%$ (no bias) or 50\% (moderate bias). Overall, BMAS performed best and is influenced the least by publication bias. However, its performance between conditions differed substantially. For example, when there was full publication bias, its performance with respect to correct $I^{2}$ evaluation (bottom left plot) differed between $28.2 \%$ correct (medium effect, small heterogeneity) and $84.7 \%$ correct (no effect, no heterogeneity). BMAS $\mathrm{S}_{\text {sig }}$ again performed worst when there was no or little bias. Its performance slightly increased when there was more publication bias, since it had more data available in those conditions. 
proportion correct

heterogeneity proportion correct

heterogeneity $>75 \%$

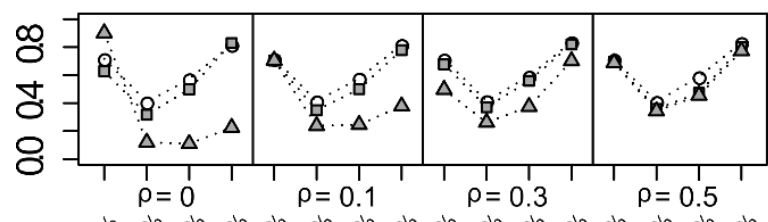

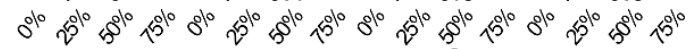
heterogeneity $\left(I^{2}\right)$

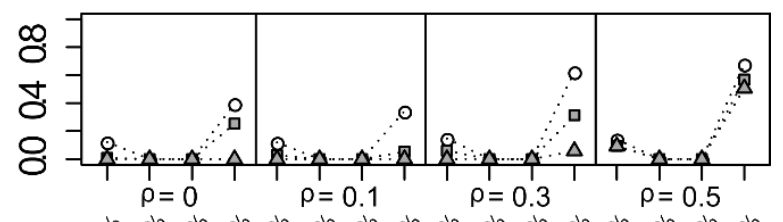

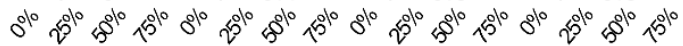
heterogeneity $\left(I^{2}\right)$

$50 \%$ publication bias

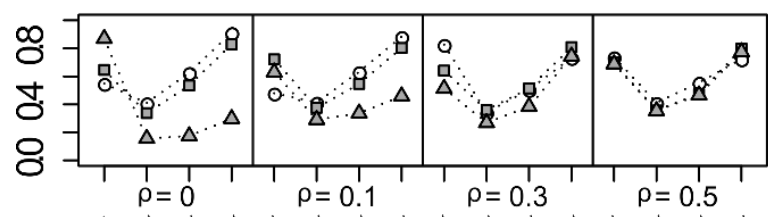

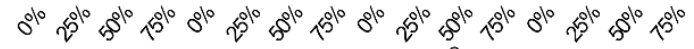
heterogeneity $\left(I^{2}\right)$

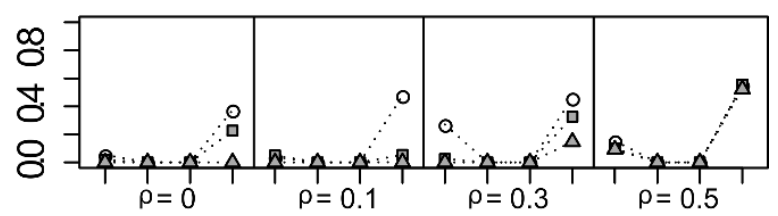

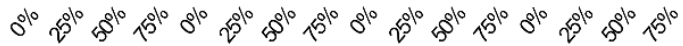
heterogeneity $\left({ }^{2}\right)$

95\% publication bias

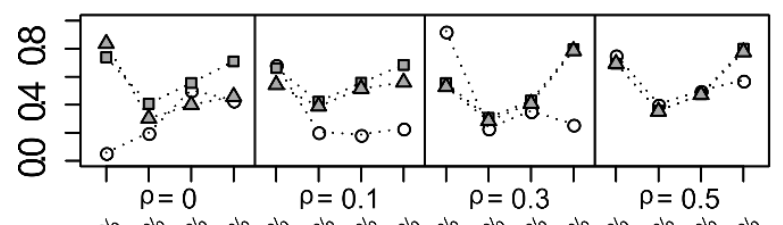

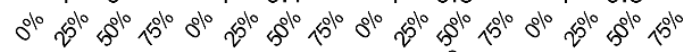
heterogeneity $\left(\mathrm{I}^{2}\right)$

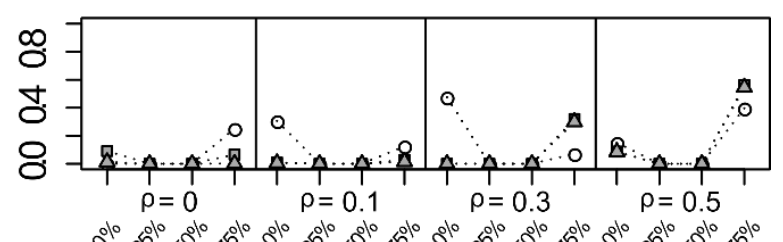

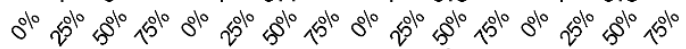
heterogeneity $\left(I^{2}\right)$

$100 \%$ publication bias

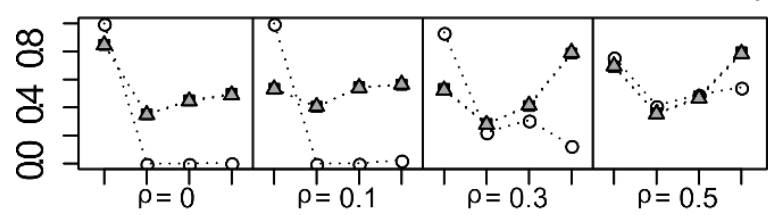

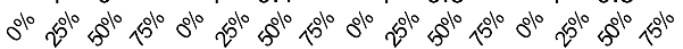
heterogeneity $\left(I^{2}\right)$

o Uncorrected BMAS

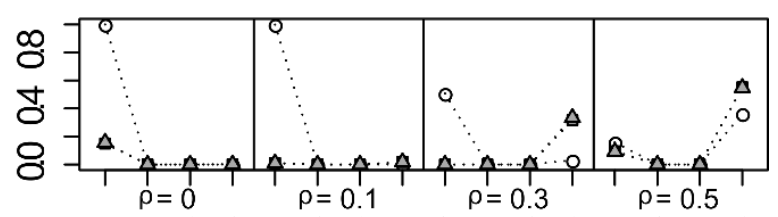

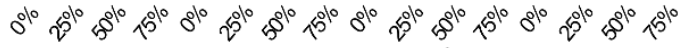
heterogeneity $\left(\mathrm{I}^{2}\right)$

$\triangle$ BMAS $_{\text {sig }}$

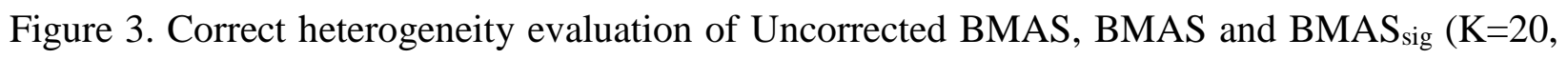
$\mathrm{N}=62$ ). The $y$-axis shows the proportion of correct heterogeneity evaluations (left) and correct evaluations with a posterior probability of at least $75 \%$ (right), based on a uniform prior, for publication bias (rows) and true effect size and heterogeneity ( $x$-axis).

\section{Performance BMAS: average effect size and effect size heterogeneity together}

Figure 4 shows the performance of the three methods when the simultaneous identification of both average effect size and heterogeneity was correct. Naturally, performance on both effect size and heterogeneity will always result in lower performance than on these factors alone. 
The pattern of results was similar as for the separate evaluations of effect sizes and heterogeneity. Uncorrected BMAS had clear problems when publication bias was present, whereas the performance of BMAS was best. When publication bias was absent (top left plot), BMAS performed slightly worse than Uncorrected BMAS, but it outperformed Uncorrected BMAS when publication bias was present. BMAS $_{\text {sig }}$ had problems when there was no publication bias, due to the aforementioned reasons. When there were 20 studies included in each meta-analysis, there were usually not sufficient data to reach relative certainty $(>75 \%)$ in the (correct) model choice for either of the methods (right column). 
proportion correct model proportion correct

model $>75 \%$

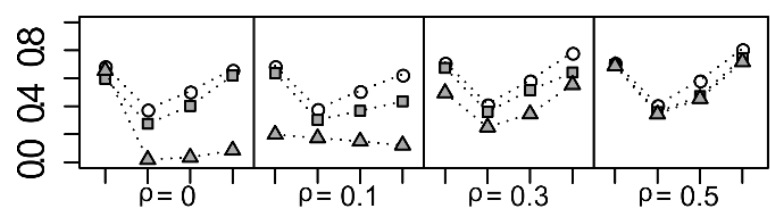

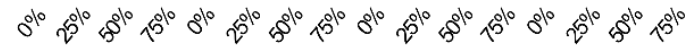
heterogeneity $\left({ }^{2}\right)$
$0 \%$ publication bias

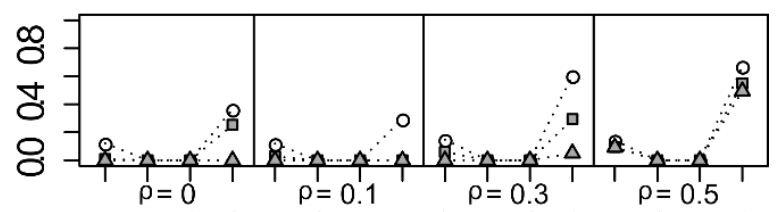

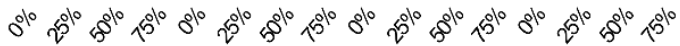
heterogeneity $\left({ }^{2}\right)$

$50 \%$ publication bias

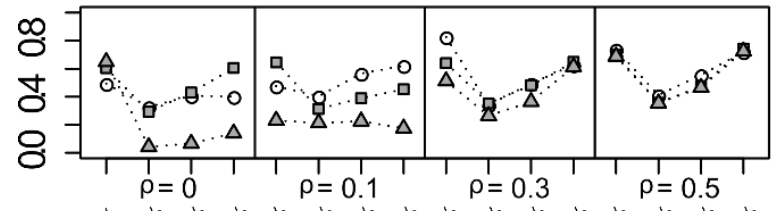

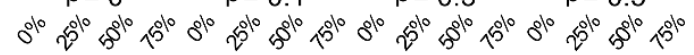
heterogeneity $\left(\mathrm{I}^{2}\right)$

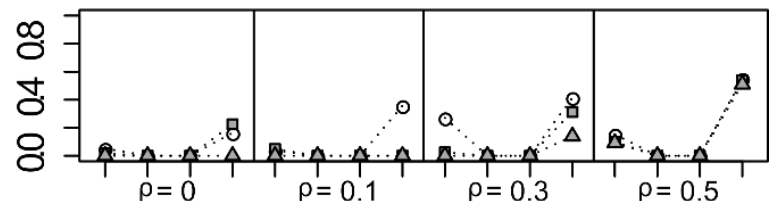

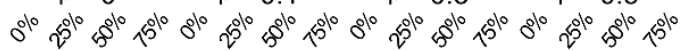
heterogeneity $\left(\mathrm{I}^{2}\right)$

95\% publication bias

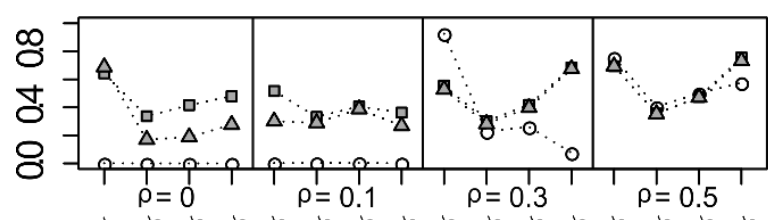

की० की० की heterogeneity $\left(\mathrm{I}^{2}\right)$

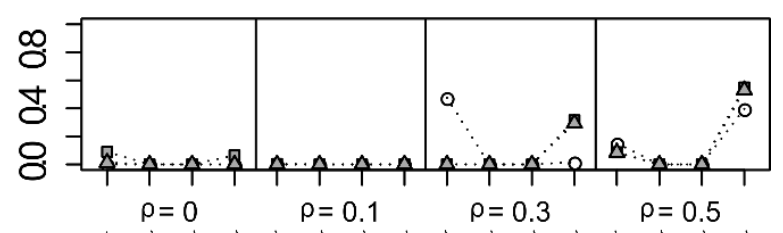

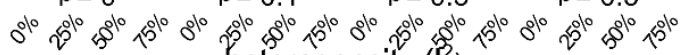
heterogeneity $\left(l^{2}\right)$

$100 \%$ publication bias

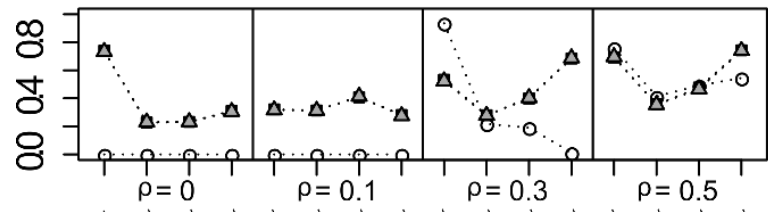

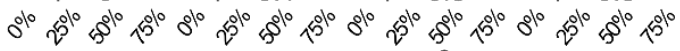
heterogeneity $\left(\mathrm{I}^{2}\right)$

๑ BMAS

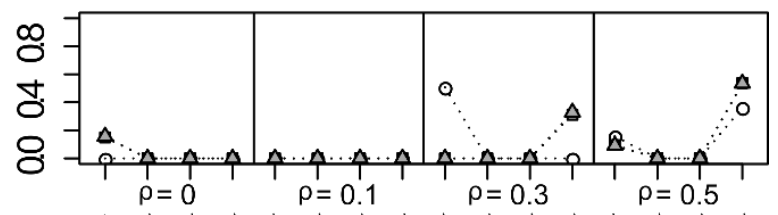

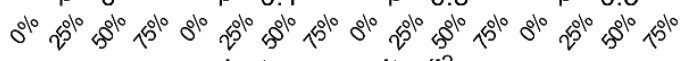
heterogeneity $\left(I^{2}\right)$

$\triangle$ BMAS $_{\text {sig }}$

Figure 4. Correct effect size and heterogeneity evaluation (correct model) of Uncorrected BMAS, BMAS and BMAS $\mathrm{Big}_{\mathrm{sig}}(\mathrm{K}=20, \mathrm{~N}=62)$. The $y$-axis shows the proportion of correct effect size and heterogeneity evaluations (left) and correct evaluations with a posterior probability of at least $75 \%$ (right), based on a uniform prior, for different publication bias (rows) and true effect size and heterogeneity (x-axis).

\section{Large meta-analytical datasets $(\mathrm{K}=100)$}

\section{Random-effects meta-analysis}


Table 7 provides the results of random effects meta-analysis when there were 100 studies within each meta-analysis. Here we do not see large differences in the point estimates of the effect size compared to the results when there were 20 studies within each meta-analysis. Hence, including more studies did not result in better estimates for the random effects meta-analysis, if publication bias was present. In contrast, including more studies will result in higher precision of the biased effect size estimate, that is, smaller confidence intervals around this incorrect estimate (Nuijten et al., 2015).

Comparing the estimates of heterogeneity for $K=20$ and $K=100$, bias was only smaller when there was no publication bias. For example, when there was no publication bias and there was medium heterogeneity, the average bias of the $I^{2}$ estimate dropped from $-0.11(0.19)$ to -0.02 (0.08) on average, for the four effect size values. When publication bias was present including more studies on average results in smaller confidence intervals around the $I^{2}$-estimate, but the estimates generally did not improve when additional data was available. For example, when there was full publication bias, no effect and large heterogeneity, heterogeneity was still estimated to be close to zero (average bias $-73 \%$ ). To conclude, effect size and heterogeneity estimates of the random effects meta-analysis only benefitted from the additionally available data when there was no publication bias.

Table 7. Average bias in effect size estimate $(\rho)$ and heterogeneity $\left(\mathrm{I}^{2}\right)$ of random effects metaanalysis, $\mathrm{K}=100, \mathrm{~N}=62$ (standard deviations between brackets)

\begin{tabular}{|c|c|c|c|c|c|c|c|c|}
\hline & \multicolumn{4}{|c|}{ Bias in $\rho$} & \multicolumn{4}{|c|}{ Bias in $\mathrm{I}^{2}$} \\
\hline & $\rho=0$ & $\rho=0.1$ & $\rho=0.3$ & $\rho=0.5$ & $\rho=0$ & $\rho=0.1$ & $\rho=0.3$ & $\rho=0.5$ \\
\hline & \multicolumn{8}{|c|}{ 0\% publication bias } \\
\hline$I^{2}=0 \%$ & $0.00(0.01)$ & $0.00(0.01)$ & $0.00(0.01)$ & $0.00(0.01)$ & $0.04(0.06)$ & $0.04(0.06)$ & $0.04(0.06)$ & $0.04(0.06)$ \\
\hline $\mathrm{I}^{2}=25 \%$ & $0.00(0.02)$ & $0.00(0.01)$ & $0.00(0.01)$ & $0.00(0.01)$ & $-0.03(0.11)$ & $-0.03(0.11)$ & $-0.03(0.11)$ & $-0.03(0.11)$ \\
\hline $\mathrm{I}^{2}=50 \%$ & $0.00(0.02)$ & $0.00(0.02)$ & $0.00(0.02)$ & $0.00(0.01)$ & $-0.02(0.07)$ & $-0.03(0.08)$ & $-0.02(0.08)$ & $-0.02(0.08)$ \\
\hline \multirow[t]{2}{*}{$\mathrm{I}^{2}=75 \%$} & $0.00(0.03)$ & $0.00(0.03)$ & $0.00(0.02)$ & $0.00(0.02)$ & $-0.01(0.04)$ & $-0.01(0.04)$ & $-0.01(0.04)$ & $-0.01(0.04)$ \\
\hline & \multicolumn{8}{|c|}{$\mathbf{5 0 \%}$ publication bias } \\
\hline $\mathrm{I}^{2}=0 \%$ & $0.01(0.01)$ & $0.03(0.01)$ & $0.02(0.01)$ & $0.00(0.01)$ & $0.12(0.10)$ & $0.11(0.09)$ & $0.01(0.02)$ & $0.03(0.06)$ \\
\hline $\mathrm{I}^{2}=25 \%$ & $0.02(0.02)$ & $0.04(0.02)$ & $0.03(0.01)$ & $0.00(0.01)$ & $0.09(0.09)$ & $0.06(0.09)$ & $-0.15(0.10)$ & $-0.06(0.10)$ \\
\hline$I^{2}=50 \%$ & $0.03(0.02)$ & $0.05(0.02)$ & $0.03(0.01)$ & $0.01(0.01)$ & $0.06(0.06)$ & $0.03(0.07)$ & $-0.11(0.10)$ & $-0.06(0.08)$ \\
\hline \multirow[t]{2}{*}{$\mathrm{I}^{2}=75 \%$} & $0.06(0.03)$ & $0.07(0.03)$ & $0.05(0.02)$ & $0.02(0.02)$ & $0.02(0.03)$ & $0.00(0.04)$ & $-0.05(0.05)$ & $-0.05(0.04)$ \\
\hline & \multicolumn{8}{|c|}{$95 \%$ publication bias } \\
\hline$I^{2}=0 \%$ & $0.13(0.02)$ & $0.14(0.01)$ & $0.04(0.01)$ & $0.00(0.01)$ & $0.37(0.08)$ & $0.00(0.02)$ & $0.00(0.00)$ & $0.03(0.05)$ \\
\hline & $0.17(0.02)$ & $0.16(0.01)$ & $0.06(0.01)$ & $0.00(0.01)$ & $0.17(0.09)$ & $-0.23(0.05)$ & $-0.25(0.01)$ & $-0.08(0.10)$ \\
\hline $\mathrm{I}^{2}=50 \%$ & $0.21(0.02)$ & $0.18(0.01)$ & $0.08(0.01)$ & $0.01(0.0$ & $-0.02(0.1$ & $-0.37(0.13)$ & $-0.42(0.10)$ & $-0.11(0.08)$ \\
\hline \multirow[t]{2}{*}{$\mathrm{I}^{2}=75 \%$} & $0.28(0.02)$ & $0.23(0.02)$ & $0.12(0.02)$ & $0.03(0.02)$ & $-0.14(0.09)$ & $-0.27(0.12)$ & $-0.26(0.09)$ & $-0.11(0.05)$ \\
\hline & \multicolumn{8}{|c|}{$100 \%$ publication bias } \\
\hline$I^{2}=0 \%$ & $0.26(0.00)$ & $0.18(0.01)$ & $0.05(0.01)$ & $0.00(0.01)$ & $0.00(0.00)$ & $0.00(0.00)$ & $0.00(0.00)$ & $0.02(0.05)$ \\
\hline$I^{2}=25 \%$ & $0.27(0.01)$ & $0.19(0.01)$ & $0.06(0.01)$ & $0.00(0.01)$ & $-0.25(0.00)$ & $-0.25(0.00)$ & $-0.25(0.00)$ & $-0.09(0.10)$ \\
\hline
\end{tabular}




\begin{tabular}{lllllllll}
\hline $\mathrm{I}^{2}=50 \%$ & $0.30(0.01)$ & $0.22(0.01)$ & $0.08(0.01)$ & $0.01(0.01)$ & $-0.50(0.00)$ & $-0.50(0.00)$ & $-0.46(0.06)$ & $-0.11(0.08)$ \\
$\mathrm{I}^{2}=75 \%$ & $0.35(0.01)$ & $0.27(0.01)$ & $0.13(0.01)$ & $0.04(0.02)$ & $-0.73(0.05)$ & $-0.65(0.11)$ & $-0.33(0.09)$ & $-0.11(0.05)$ \\
\hline
\end{tabular}

\section{Performance BMAS: average effect size}

Figure 5 shows the performance of the three methods concerning the evaluation of average effect size. What is striking is that the methods rarely had a probability of at least 0.8 to assign more than $75 \%$ certainty (corresponding to BMAS factor > 3) to the correct average effect size. Hence, even 100 studies were often not enough to reliably distinguish zero, small, medium, and large true average effect size. However, BMAS and Uncorrected BMAS did often correctly identify the correct model, in certain conditions. We discuss their performance in some detail, but not $\mathrm{BMAS}_{\mathrm{sig}}$ as it never performs better than BMAS.

Uncorrected BMAS showed the best performance in case of no publication bias, with at least $97.1 \%$ correct identification of the correct true effect size (top left subfigure). For increasing publication bias Uncorrected BMAS performed worse than when 20 studies were included. The Uncorrected BMAS hardly ever identified the correct model in case of strong publication bias (95\%-100\%) in combination with a zero or small true effect size ( $\max 0.03 \%$ correct). Uncorrected BMAS did however assign high posterior probabilities to the (incorrect) models in these conditions (average posterior probability of .911, results not shown). This false certainty is comparable to the narrow confidence intervals of random-effects meta-analysis around the biased effect size estimate. For the other conditions (strong publication bias in combination with medium and large true effect size, and 50\% publication bias), the performance of Uncorrected BMAS was similar to that of BMAS, with some exceptions at high heterogeneity $\left(I^{2}=0.75\right)$ where BMAS performed best.

BMAS performed best in the publication bias conditions. Over all 64 conditions, it identified the correct effect size in $93.7 \%$ of all iterations (standard deviation between conditions $=10.5 \%$ ). Correct identification was at least $52.9 \%$, which occurred for $100 \%$ publication bias in combination with a true zero effect size and medium heterogeneity. BMAS reached the $>75 \%$ certainty threshold for the correct effect size most often (63.7\% of all iterations, standard deviation between conditions $=23.2 \%$ ) Strikingly, BMAS hardly ever assigned more than $75 \%$ probability to the correct model under $100 \%$ publication bias in combination with zero true effect size and small or medium heterogeneity (5\% and $4 \%$ respectively), or small true effect size with zero or small heterogeneity $(0.3 \%$ and $0.8 \%)$, even though our consistency simulation studied showed that 
BMAS is in fact consistent in these conditions. This demonstrates that under extreme publication bias one needs many data points to reliably identify the correct model.

\section{Performance BMAS: effect size heterogeneity}

Figure 6 shows the performance of the three methods concerning the evaluation of effect size heterogeneity. The same patterns can be seen as for 20 studies. BMAS performed better and reached $>75 \%$ certainty (right column) for the correct heterogeneity model more often, whereas Uncorrected BMAS performed better in some of the conditions, but worse in others. We will again discuss the performance of the methods in some detail, except for BMAS $S_{\text {sig, }}$ since it never outperforms BMAS.

In case of no publication bias, Uncorrected BMAS performed best and had more certainty in its correct heterogeneity estimate. It reached the $>75 \%$ certainty threshold for the correct heterogeneity model in $75.3 \%$ of the 16 no publication bias conditions (top right figure), whereas this was only $16.1 \%$ when there were 20 studies available. When publication bias increased, the performance of Uncorrected BMAS decreased. Whereas it identified the correct heterogeneity model correct in $87.9 \%$ of the no-publication bias conditions, this dropped to $77.4 \%$ when there was $50 \%$ publication bias, $45.1 \%$ when there was $95 \%$ publication bias and $39.6 \%$ in case of full publication bias. Uncorrected BMAS actually only performed well when true heterogeneity was $0 \%$, since it often estimated heterogeneity to be $0 \%$, in the face of full publication bias, regardless of its true value. This is due to the fact that when there is full publication bias, the remaining studies often show extreme homogeneity $\left(I^{2}<0 \%\right)$, see also Augusteijn, et al (2019). Since $I^{2}=0 \%$ is the model with the lowest amount of heterogeneity, its evidence accumulates in this model.

The performance of BMAS on the other hand was relatively stable in its evaluation of heterogeneity. In most conditions, its performance actually slightly increased when publication bias was present, compared to the conditions without publication bias. Only when there was full publication bias (figures on last row), its performance decreased. Compared to the case of 20 studies, BMAS reached the $75 \%$ certainty threshold more often. In case of no publication bias, BMAS had an average probability of .85 to evaluate heterogeneity correctly (.66 with $>75 \%$ certainty). When there was $50 \%$ or $95 \%$ bias, these average probabilities were $.85(.66>75 \%)$ and $.85(.64>75 \%)$, respectively. When there was full publication bias, the heterogeneity was correctly evaluated with a probability of.78 (.46> 75\% certainty).

Performance BMAS: average effect size and effect size heterogeneity together 
Finally, Figure 7 shows the results of the three methods using the strictest criteria: having both the effect size and heterogeneity correct. Unsurprisingly, we see the same patterns; compared to 20 studies, BMAS performed better and reached $>75 \%$ certainty (right column) more often, whereas Uncorrected BMAS only performed better in conditions where it already performed relatively well.

Uncorrected BMAS performed best when there was no publication bias, with an average probability of .88 to evaluate the model correctly. Performance was decreasing in publication bias, with correct evaluations (average posterior probabilities) equal to $71.4 \%$ (50\% bias), $28.1 \%$ (95\% bias) and $26.9 \%$ (full publication bias). In case of substantial publication bias (95 or 100\%) Uncorrected BMAS only performed reasonably well when most primary studies were statistically significant (i.e., if true effect size was large, or when the effect size was medium in combination with no heterogeneity); in the other 22 substantial publication bias conditions, the probability of correct model evaluation was only .04.

BMAS performed relatively well and evaluated the correct model in $80.7 \%$ of the 64 conditions (ranging from 51.8-98.1\%), and did so with $>75 \%$ certainty in $59.5 \%$ of the iterations. In case of full publication bias, performance of BMAS was not sufficient; the average probability of correct model evaluation was $74 \%$ (minimum $51.8 \%$, when $\rho=0, I^{2}=50 \%$ ), and the average probability of correct model evaluation with a certainty $>75 \%$ was only $45 \%$ with large variations between conditions (ranging from $0.3 \%-97 \%$, standard deviation $=33 \%$ ). Hence, 100 studies was still not enough for reliable and precise model evaluation when there was full publication bias. 


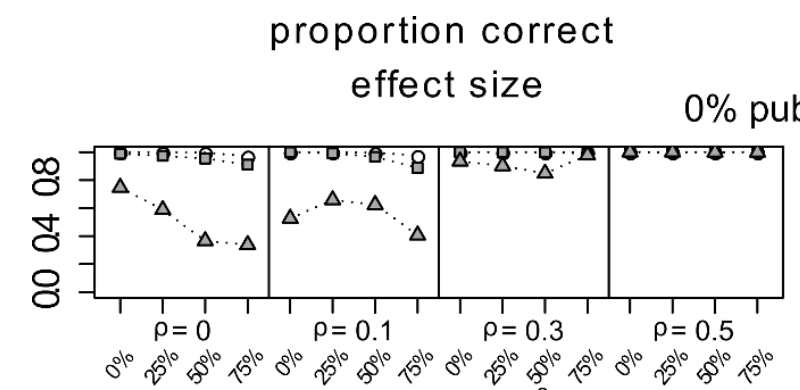

heterogeneity $\left(1^{2}\right)$ proportion correct effect size $>75 \%$
$50 \%$ publication bias

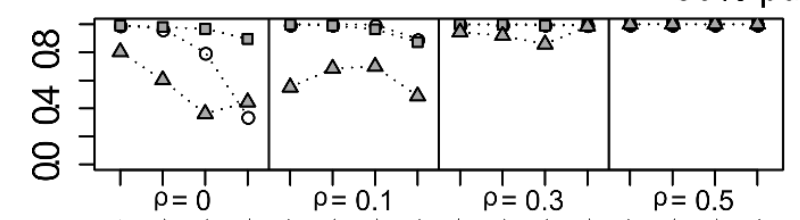

की० heterogeneity $\left(\mathrm{I}^{2}\right)$

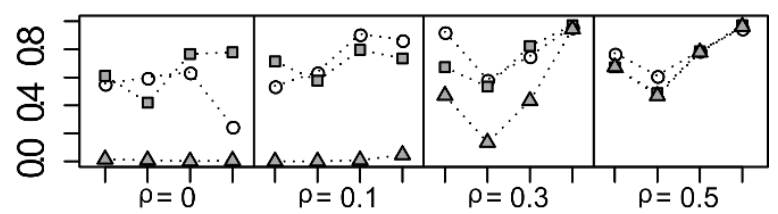

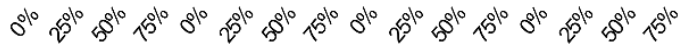
heterogeneity $\left(I^{2}\right)$

95\% publication bias

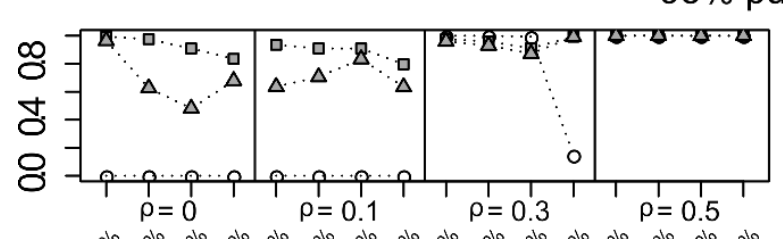

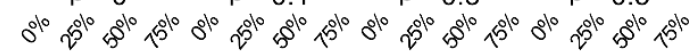
heterogeneity $\left(\mathrm{I}^{2}\right)$

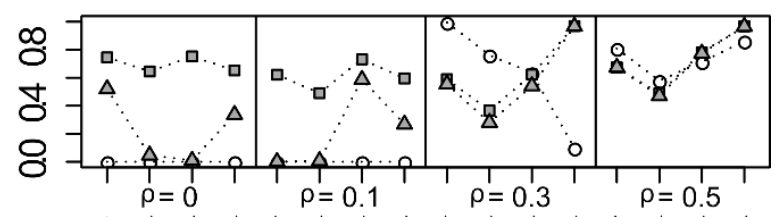

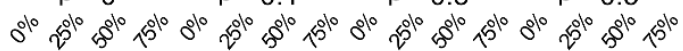
heterogeneity $\left(\mathrm{I}^{2}\right)$

$100 \%$ publication bias

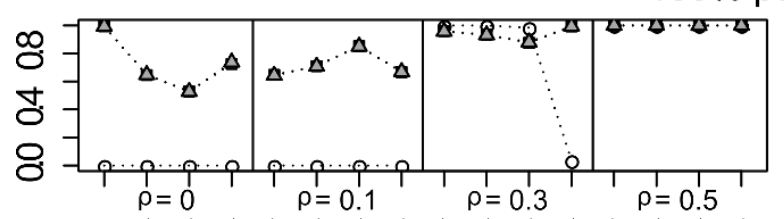

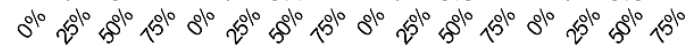
heterogeneity $\left(I^{2}\right)$

o Uncorrected BMAS
- BMAS

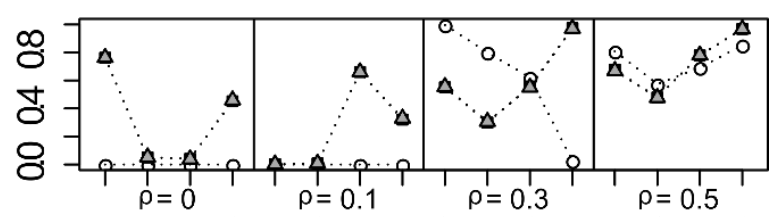
वे की० heterogeneity $\left(I^{2}\right)$

$\triangle$ BMAS $_{\text {sig }}$

Figure 5. Correct effect size evaluation of Uncorrected BMAS, BMAS and BMAS ${ }_{\text {sig }}$ in large datasets $(K=100, N=62)$. Y-axis shows the proportion of correct effect size evaluations (left) and correct evaluations with a posterior probability of at least $75 \%$ (right), based on a uniform prior, for different levels of publication bias (plot rows) and different levels of the true effect size and levels of heterogeneity (X-axis). 
proportion correct

heterogeneity proportion correct

heterogeneity $>75 \%$

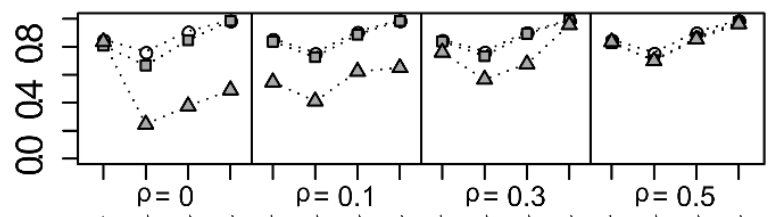

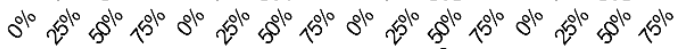
heterogeneity $\left(1^{2}\right)$

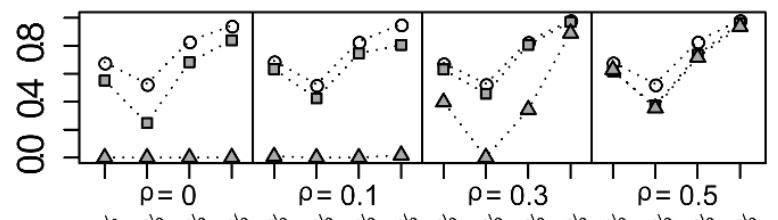

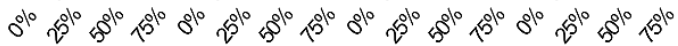
heterogeneity $\left(1^{2}\right)$

$50 \%$ publication bias

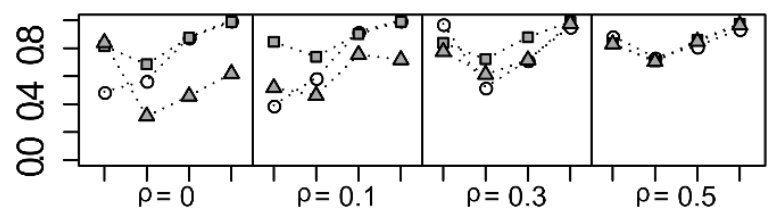

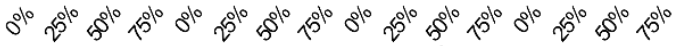
heterogeneity $\left(1^{2}\right)$

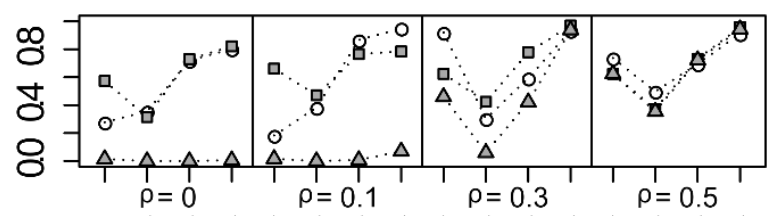

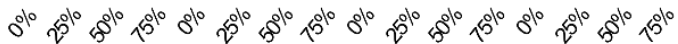
heterogeneity $\left(1^{2}\right)$

$95 \%$ publication bias

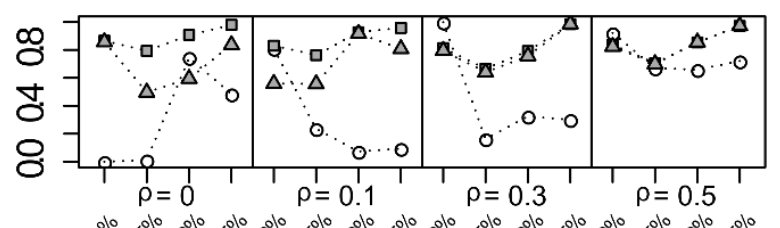

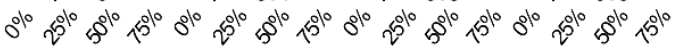
heterogeneity $\left(1^{2}\right)$

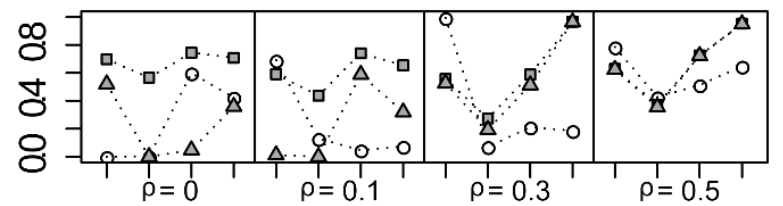

ग10 heterogeneity $\left({ }^{2}\right)$

$100 \%$ publication bias

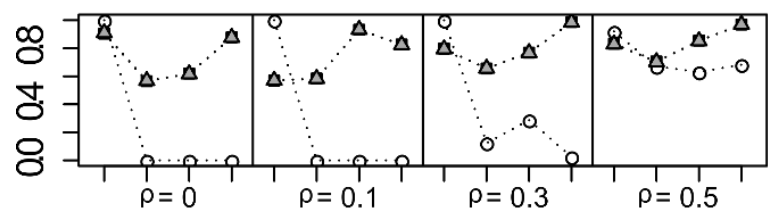

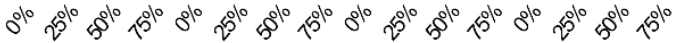
heterogeneity $\left(1^{2}\right)$

- Uncorrected BMAS

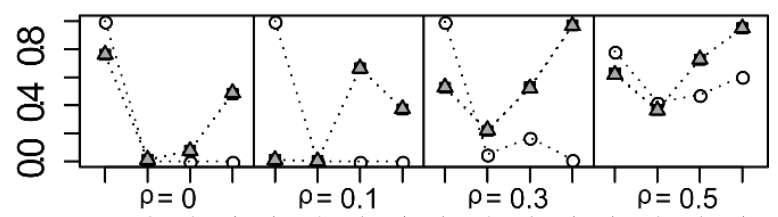

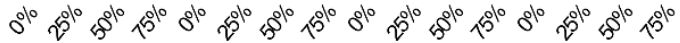
heterogeneity $\left(1^{2}\right)$

$\triangle$ BMAS sig

Figure 6. Correct heterogeneity evaluation of Uncorrected BMAS, BMAS and BMAS $\mathrm{sig}_{\text {ig }}$ in large data-sets ( $\mathrm{K}=100, \mathrm{~N}=62)$. Y-axis shows the proportion of correct heterogeneity evaluations (left) and correct evaluations with a posterior probability of at least $75 \%$ (right), based on a uniform prior, for different levels of publication bias (plot rows) and different levels of the true effect size and levels of heterogeneity (X-axis). 
proportion correct

proportion correct

model

$0 \%$ publication bias

model $>75 \%$

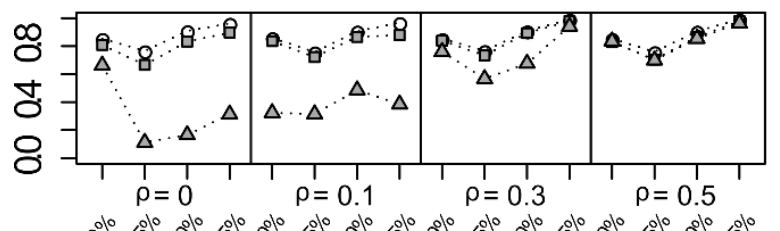

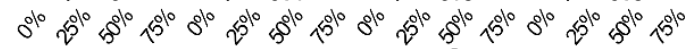
heterogeneity $\left(I^{2}\right)$

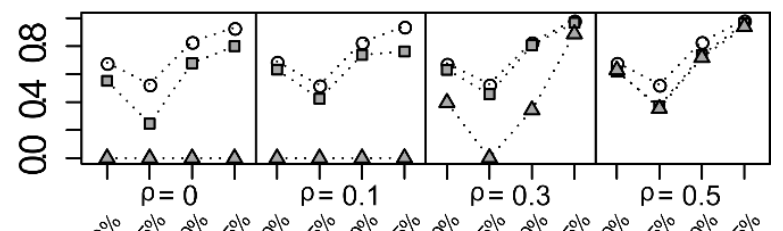

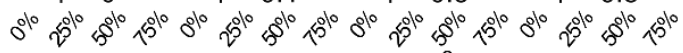
heterogeneity $\left(1^{2}\right)$

$50 \%$ publication bias

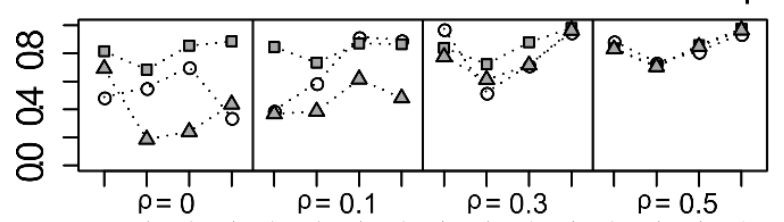

ग0 heterogeneity $\left(1^{2}\right)$

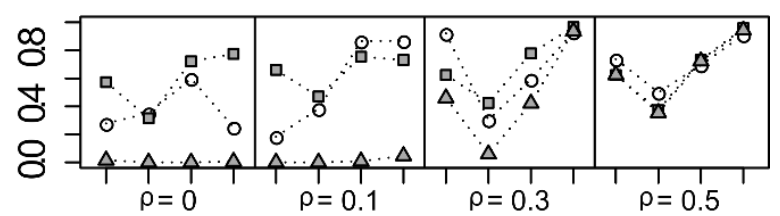

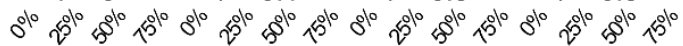
heterogeneity $\left(1^{2}\right)$

$95 \%$ publication bias

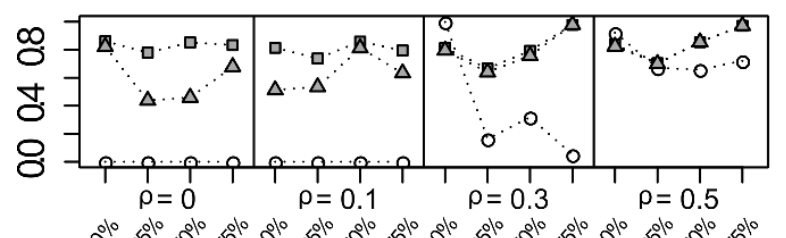

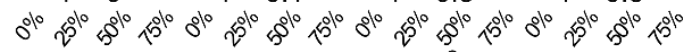
heterogeneity $\left(1^{2}\right)$

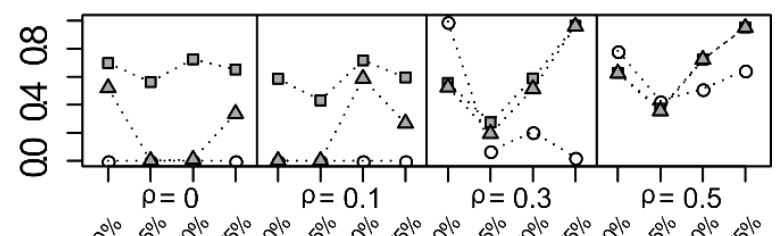

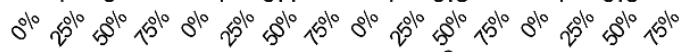
heterogeneity $\left(1^{2}\right)$

$100 \%$ publication bias

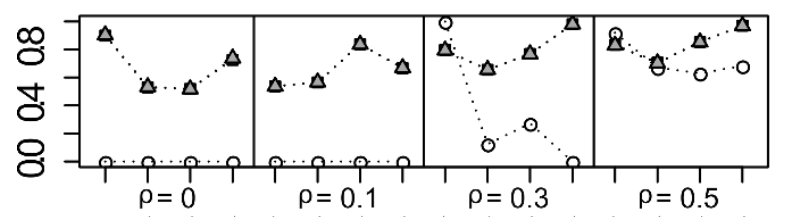

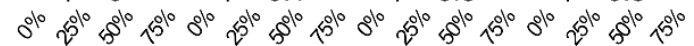
heterogeneity $\left(I^{2}\right)$

○ Uncorrected BMAS

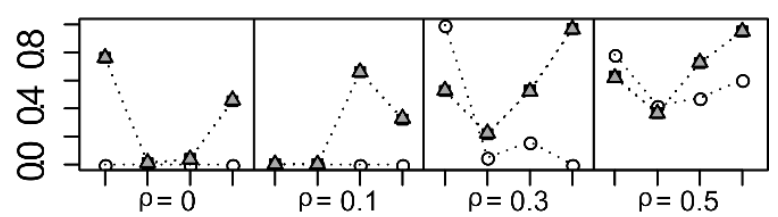

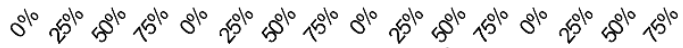
heterogeneity $\left({ }^{2}\right)$

口 BMAS

$\triangle$ BMAS sig

Figure 7. Correct effect size and heterogeneity evaluation (correct model) of Uncorrected BMAS, BMAS and BMAS $S_{\text {sig }}$ in large data-sets $(K=100, N=62)$. Y-axis shows the proportion of correct effect size and heterogeneity evaluations (left) and correct evaluations with a posterior probability of at least 75\% (right), based on a uniform prior, for different levels of publication bias (plot rows) and different levels of the true effect size and levels of heterogeneity (X-axis). 


\section{Small meta-analysis $(K=5)$}

The supplemental materials provide the results for five studies $(\mathrm{K}=5)$, representing a small meta-analysis. Posterior probabilities for the correct model (both effect size and heterogeneity correct) hardly ever exceeded 0.75 with Uncorrected BMAS under no publication bias (maximum .17), and with BMAS under publication bias (maximum .15). Even correct identification of these methods, without the $75 \%$ certainty requirement, was low (Uncorrected BMAS $25.8 \%$ on average [min $0 \%$, max $82.6 \%$ ], BMAS $27.0 \%$ on average [min. $7.4 \%$, max. 66.6\%].). To conclude, metaanalyses with five or less small studies (with $N=62$ ) do not allow for precise inferences on effect size and effect size heterogeneity.

\section{Application}

We applied all three versions of BMAS method to a meta-analysis by McCall and Carriger (1993), containing 31 samples on infant habituation and recognition memory performance as predictors of later IQ. The sample sizes of these 31 studies differed from 11 until 143, and the effect sizes differed between $r=.01$ and $r=.66$. Twenty-six of these primary study effect sizes were statistically significant when tested one-sided with $\alpha=0.05$ (84\%), 28 of them were statistically significant if tested two-sided (90\%). A random effects meta-analysis ${ }^{1}$ shows that there was a medium to large effect: $r=.407, p<.0001,95 \% \mathrm{CI}$ ranged from .341 to .469]. The $Q$-test of homogeneity $(Q(30)=49.57, p=.0137)$ indicated small to medium heterogeneity $\left(I^{2}=39.5 \%\right.$ $[2.6 \%: 55.3 \%])$.

Table 8 shows the results of all three BMAS methods for the McCall and Carriger (1993) dataset, using a uniform prior. Uncorrected BMAS (top of Table 8) suggests a large effect (BMAS Factor $=2.9)$ and strongly suggests medium heterogeneity $($ BMAS Factor $=6.1)$, which is in line with the results of random effects meta-analysis. There is some, but not convincing evidence for the combination of medium heterogeneity and a large effect size $($ BMAS Factor $=2.3)$.

Table 8. Posterior probabilities of Uncorrected BMAS, BMAS and BMASs $\mathrm{Bg}_{\mathrm{ig}}$ for McCall and Carriger (1993), using a uniform prior.

\begin{tabular}{lllcll}
\hline & $\rho=0$ & $\rho=0.1$ & $\rho=0.3$ & $\rho=0.5$ & \\
$I^{2}=0 \%$ & 0 & 0 & Uncorrected BMAS & & \\
\hline
\end{tabular}

\footnotetext{
${ }^{1}$ Random effects meta-analysis was conducted with use of the Metafor package (Viechtbauer, 2010), using the DerSimonian-Laird estimator on fisher transformed correlations. Confidence intervals of heterogeneity were determined with use of the Q-profile method (Viechtbauer, 2007).
} 


\begin{tabular}{lllcll}
$I^{2}=25 \%$ & 0 & 0 & 0.080 & 0.010 & 0.089 \\
$I^{2}=50 \%$ & 0 & 0 & 0.166 & $\mathbf{0 . 6 9 3}$ & $\mathbf{0 . 8 5 9}$ \\
$I^{2}=75 \%$ & 0 & 0 & 0.007 & 0.043 & 0.049 \\
& 0 & 0 & 0.254 & $\mathbf{0 . 7 4 6}$ & \\
$I^{2}=0 \%$ & 0 & & $\mathbf{B M A S}$ & & \\
$I^{2}=25 \%$ & 0 & 0 & 0.020 & 0 & 0.020 \\
$I^{2}=50 \%$ & $2 \mathrm{e}-04$ & 0 & 0.335 & 0 & 0.335 \\
$I^{2}=75 \%$ & 0.040 & 0.072 & $\mathbf{0 . 5 0 2}$ & $1 \mathrm{e}-04$ & $\mathbf{0 . 5 1 7}$ \\
& 0.040 & 0.086 & $\mathbf{0 . 8 7 3}$ & $2 \mathrm{e}-04$ & 0.129 \\
$I^{2}=0 \%$ & 0 & & $\mathbf{B M A S}$ sig & & \\
$I^{2}=25 \%$ & 0 & 0 & 0.088 & 0 & 0.088 \\
$I^{2}=50 \%$ & $5 \mathrm{e}-04$ & 0 & 0.302 & $1 \mathrm{e}-04$ & 0.302 \\
$I^{2}=75 \%$ & 0.123 & 0.017 & $\mathbf{0 . 3 2 3}$ & $1 \mathrm{e}-04$ & $\mathbf{0 . 3 4 0}$ \\
& 0.124 & 0.131 & 0.015 & 0 & 0.270 \\
\hline
\end{tabular}

When correcting for the possible presence of publication bias with BMAS (middle of Table 8), a large effect size was deemed very unlikely (posterior probability of .0002, which is 3,730 less likely than under Uncorrected BMAS). Instead a medium effect size was considered to be most probable $($ BMAS Factor $=6.9)$, whereas a zero true effect was deemed unlikely (posterior probability equal to .04). Medium heterogeneity still received most, but weak support (BMAS Factor $=1.07)$. Medium effect size in combination with medium heterogeneity were considered most likely, but did not receive strong support $($ BMAS Factor $=1.01)$.

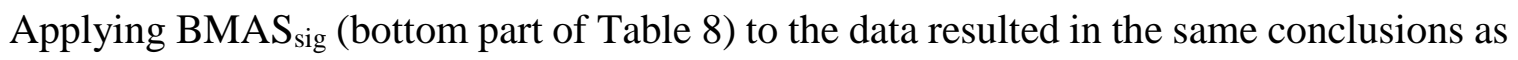
when applying BMAS; most evidence in favor of a medium effect and medium heterogeneity. The evidence and posterior probabilities were slightly lower than for BMAS as it only uses the 28 statistically significant effect sizes rather than the complete set of 31 studies.

Comparing the results of the three methods provides information on the presence of publication bias. If the results of the three methods are similar, that is, if all three methods yield most support for the same models, then publication bias is unlikely present or does not affect the conclusions of the meta-analysis. In the example of McCall and Carriger the considerably attenuated evaluation of average effect size suggests that publication bias was present and that one should try to correct for it when evaluating average effect size and effect size heterogeneity.

\section{Shiny app: BMAS}


A web application (https://augusteijn.shinyapps.io/BMAS/) is available to apply all three different methods (uncorrected BMAS, BMAS and BMAS ${ }_{\text {sig }}$ ) to the meta-analytic data. The user can upload their own dataset, as well as apply the methods to the example dataset of McCall and

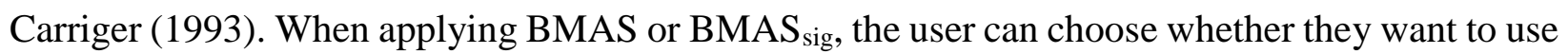
one- or two-tailed testing and can choose their $\alpha$-level. By default, this is set to one-tailed testing with $\alpha=.05$. Finally, all three priors are available: the uniform prior, the null prior, and the normal prior, with default values or values inserted by the user.

The web application provides the results of the random effects meta-analysis, as well as the method chosen by the user. The results of BMAS are provided both in a graph and in a table and can be downloaded by the user. The graph offers a visual representation of the BMAS results: on the $x$-axis it has the four effect sizes, on the $y$-axis it has the four heterogeneity levels. The $z$ axis shows the posterior probabilities. The model with the highest posterior probability is colored green. This visual application allows the user to easily grasp the distribution of weights over the sixteen models, and whether there are large differences between them, or whether the probabilities are relatively close to each other. Figure 8 shows the layout of the BMAS web application. Here, BMAS was applied to the dataset by McCall and Carriger (1993) using a one-tailed test, $\alpha=.05$ and a uniform prior. For more details on this web application, please have a look at the manual on how to use this application, also available on OSF. 


\section{Bayesian Meta-Analytic Snapshot}

\section{Manual on how to use this application on OSF}

Paper about the BMAS method (in preparation)

Author: Hilde E.M.Augusteijn

Effect size input type
O correlation (pearson r)
Data input type (see manual)
O Example dataset
Opload csV file (see manual)
BMAS method
O BMAS uncorrected
BMAS
BMAS sig
Test one- or two-sided
One-sided
O Two-sided
Alpha
0.05

Example dataset by McCall and Carriger, 1993.

This dataset contains 31 effect sizes.

Results from Random Effects Meta-analysis show an effect size of $r=0.407$ (95Cl: 0.341:0.469), $p<.001$

There is significant heterogeneity $(Q(30)=49.574, p=.014)$.

$R^{2}=39.48 \%(95 \mathrm{Cl}=2.63 \%: 55.33 \%), T^{2}=0.017(95 \mathrm{Cl}=0.001: 0.032)$

\section{Prior type}

(- uniform prior

null-model prior (see manual)

normal distribution prior (see manual)

Go
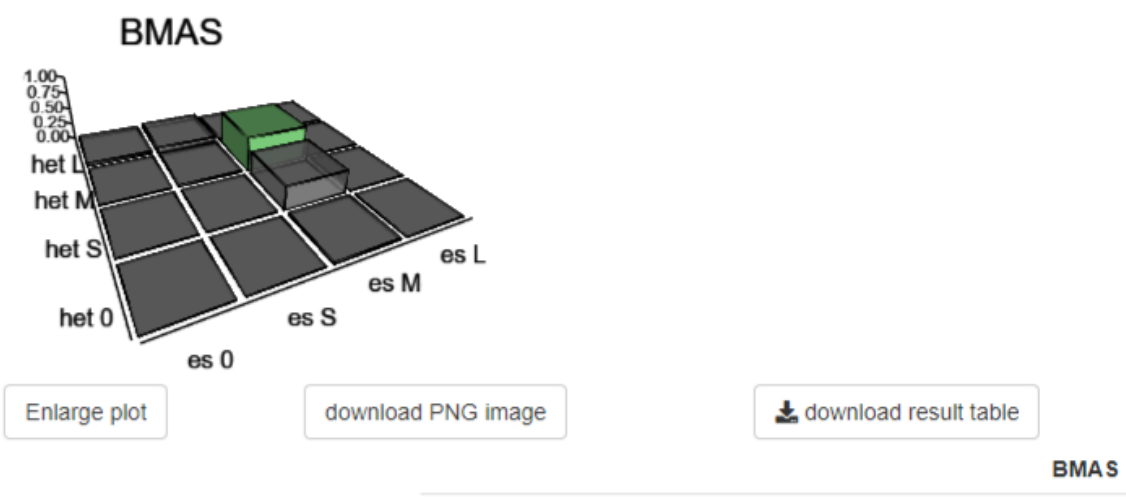

\begin{tabular}{llllll}
\cline { 2 - 4 } model & es & es & esM & esL & cummulative \\
\hline het 0 & 0 & 0 & 0.0199 & 0 & 0.0199 \\
\hline het S & 0 & 0 & 0.3349 & 0 & 0.3349 \\
\hline het $M$ & $2 \mathrm{e}-04$ & 0.0147 & 0.5015 & $1 \mathrm{e}-04$ & 0.5166 \\
\hline het L & 0.0401 & 0.0717 & 0.0167 & 0 & 0.1285 \\
\hline cummulative & 0.0403 & 0.0864 & 0.8731 & $2 \mathrm{e}-04$ & 1
\end{tabular}

Figure 8. Screenshot of sShiny BMAS web application using the example dataset of McCall and Carriger (1993), after BMAS analysis has been run, using one-tailed testing, $\alpha=.05$ and a uniform prior. 


\section{Discussion}

Publication bias remains a large threat for the validity of the results in meta-analysis, and current methods are unable to provide an evaluation of the underlying effect size and heterogeneity, which is both corrected and intuitive evaluation of the underlying effect size and heterogeneity. The method proposed in this article, Bayesian Meta-Analytic Snapshot, has some clear benefits. The methodology and the results of BMAS are intuitive. Compared to $p$-values and confidence intervals, BMAS shows the relative posterior probabilities, and thereby provides a clearer interpretation. One can easily evaluate the precision of the evaluation, by comparing multiple models with each other by looking at their posterior probabilities. If these values are close together, we have not enough evidence, or data, to distinguish between the different models. Low posterior probabilities indicate that a model is rather unlikely, whereas larger values indicate that a model, or a set of models, is likely. Furthermore, BMAS can correct for publication bias, and can provides a sensitivity analysis of the presence and influence of publication bias by comparing the Uncorrected BMAS with the BMAS or BMAS $S_{\text {sig }}$ method. Large changes in the relative posterior probabilities indicate that publication bias is present. Comparison of Uncorrected BMAS and BMAS indicates the degree of overestimation of the effect size, and its influence on the estimate of heterogeneity. A final benefit of BMAS is that it allows for an intuitive and computationally easy way of updating priors, and adding new data to the meta-analysis.

Our simulation studies showed that BMAS and BMAS sig are consistent, that is they identified the correct model in case of large number of primary study effect sizes, regardless of the presence of publication bias. Hence, BMAS and BMAS $S_{\text {sig }}$ accurately adjust for possible publication bias. BMAS $\mathrm{sig}_{\text {ig }}$ is less efficient than BMAS as it only employs the statistically significant studies, and hence often required more studies to identify the correct model with certainty. In case of full publication bias most studies were needed to converge to the correct model. This problem is not unique for BMAS, as also other meta-analytic methods have problems with estimating effect size and heterogeneity in this condition (Carter et al., 2019; McShane, et al., 2016).

Random-effects meta-analysis and Uncorrected BMAS only performed well in case of no publication bias or when most primary study effect sizes were not suppressed (i.e., if statistical power is high, and most primary study effect sizes are statistically significant). If this is not the case, results of both methods are biased, leading to overestimated effect sizes and possible severely 
under- or overestimated effect size heterogeneity. Because a true zero effect size is overestimated in case of publication bias with random effects meta-analysis and Uncorrected BMAS, we strongly recommend applying BMAS to evaluate the likelihood of a zero true effect size, to safeguard against the possibility of false positives. On the other hand, it must be noted that random-effects meta-analysis as well as Uncorrected BMAS perform best in case of no publication. Thus, if there is no evidence of publication bias we recommend focusing on interpreting the output of randomeffects meta-analysis and Uncorrected BMAS. There are multiple ways to look for evidence of publication bias. For instance, absence of publication bias or no effect of publication bias is suggested by similar results of Uncorrected BMAS and BMAS, a large proportion of statistically non-significant effect sizes (van Aert, Wicherts, van Assen, 2019), and no association between standard error and effect size of primary studies (Begg \& Mazumdar, 1994; Egger, Smith, Schneider \& Minder, 1997; Lin \& Chu, 2018).

Although BMAS and BMAS sig perform well in the limit, the simulation results show that one may need a substantial number of studies to obtain strong evidence in favor of the true effect size and effect size heterogeneity. Five studies hardly ever provided conclusive evidence, 20 studies was often enough to identify the correct model but not to obtain $>75 \%$ confidence, and although 100 studies led to very frequent correct identifications and a higher proportion of 'certainty' calls, often there was still much uncertainty to decide on one specific model. This does not mean that BMAS should not be used. It just shows that much data may be needed to obtain strong conclusions on effect size and heterogeneity. Note, however, that these conclusions were obtained using simulations with typical (rather small) samples in psychology $(\mathrm{N}=62)$. Of course, in case of much larger samples (say, $\mathrm{N}=1,000$ ), much less studies are needed to reliably evaluate average effect size.

The results reveal that BMAS $\mathrm{Big}_{\text {sig }}$ never performs better than BMAS, which makes perfect

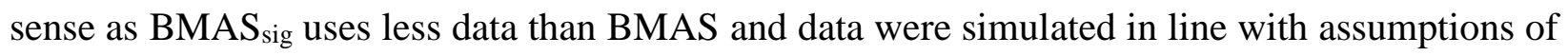
BMAS. This does not imply that we recommend never to apply BMAS ${ }_{\text {sig. }}$ BMAS $S_{\text {sig }}$ makes less assumptions than BMAS, that is, BMAS assumes that all statistically non-significant studies are equally likely to get published, whereas $\mathrm{BMAS}_{\text {sig }}$ is unaffected by these studies. Consequently, if this assumption of BMAS is strongly violated BMAS may provide less accurate estimates than BMAS $_{\text {sig }}$ (Simonsohn, Simmons \& Nelson, 2017). For example when direction of the nonsignificant effect influences the probability of publication, or if results with a $p$-value of .08 are 
more likely to be published than results with a p-value of .92. If there are reasons to believe that not all non-significant studies are equally likely to be published, or if one prefers not to make assumptions about the non-significant publication probabilities, application of $\mathrm{BMAS}_{\mathrm{sig}}$ is reasonable. We therefore recommend applying both BMAS and BMAS ${ }_{\text {sig, and caution interpreting }}$ results of BMAS in case of diverging results of both methods.

A limitation of this study is that it examines the performance of the methods using rather small and fixed sample size for all studies. As mentioned before, increasing sample size but keeping it fixed will affect the results: fewer studies are needed to correctly identify the true effect size, and effects of publication bias on the performance of random-effects meta-analysis and Uncorrected BMAS are less detrimental as more primary studies get statistically significant. However, the pattern of results is largely unaffected by changing the fixed sample size. Changing a fixed sample size to varying study sample sizes will also affect the results, particularly the results on heterogeneity. Augusteijn et al. (2019) shows that underestimation of heterogeneity in case of publication bias is much less common if study sample sizes differ then when they are the same (see the high frequency of $I^{2}=0$ of random-effects meta-analysis and Uncorrected BMAS in case of publication bias). Note though, that BMAS methods and the shiny web application just as easily can be applied to applications with varying study sample sizes.

A limitation of the BMAS methods is that it does not provide a point estimate, but only evaluates the probability of four effects sizes and four levels of heterogeneity. This may seem most problematic when true effect size is in between two values considered, for instance, if true effect size is 0.4 (exactly in between medium and large). In such cases BMAS will divide its posterior probabilities between these models, resulting in lower posterior probabilities for each of these models. We do not consider this characteristic of BMAS as a disadvantage. We believe that a point estimate of a random-effects meta-analysis is also less of interest than an interval estimate, because the interval estimate signals both the range and precision of the estimate. The posterior probabilities of the models serve the same function, and we believe are clear and easy to interpret. Another limitation is that the shiny web application of BMAS only uses correlations. The method will be extended to also work for other effect sizes such as Cohen's $d$, Hedges' $g$, and odds ratios.

A final limitation concerns the BMAS Factor. The interpretation of evidence in favor of one of the model with a requirement of BMAS Factor $>3$ (and a posterior probability of .75) may be considered quite conservative. Using a Bayes Factor of 3 may already be considered to be 
conservative, compared to using $\alpha=.05$ with the null-hypothesis significance testing approach (Jeon \& de Boeck, 2017). The threshold of BMAS factor > 3 is more conservative, since it compares 16 models instead of two. Where the Bayes Factor starts with $\mathrm{BF}_{01}=1$, indicating that both models are equally likely, the BMAS Factor model starts off with a value of 1/15, indicating that each model is equally likely. However, as many of these 16 models imply very different states of the world, some of these models do not or hardly contribute to the BMAS factor, particularly in case of more data. In our experience, the BMAS factor is considerable less than five times smaller compared to the Bayes Factor comparing the two best-fitting models. From that perspective, a BMAS factor of 3 may be compared to a Bayes Factor from 3 to 10, which are all reasonable thresholds considered in the literature (Schönbrodt \& Wagenmakers, 2018).

To conclude, this article introduced a new and intuitive meta-analysis method, BMAS, that (i) shows which effect size and heterogeneity model are most likely given the data, (ii) provides insight in the level of (un)certainty of effect size and heterogeneity, (iii) adjust for possible publication bias, and (iv) allows for sensitivity analysis with respect to possible publication bias. A Shiny web application allows researchers to easily apply this method when conducting a metaanalysis. We would like to end with some practical recommendations for researchers conducting a meta-analysis with BMAS. When conducting a meta-analysis apply standard (fixed-effect or random-effects) meta-analysis, in accordance with the Meta-Analysis Reporting Standards (MARS; American Psychological Association, 2008), and accompany these with results from three BMAS methods:

1. Be reluctant to interpret the results of the standard meta-analysis, particularly the result of the test of the average effect, if both BMAS suggests a lower effect size than Uncorrected BMAS and BMAS considers a null effect to be likely;

2. Use BMAS rather than standard meta-analysis when expecting or wanting to report evidence in favor of the null-hypothesis;

3. Report the results of BMAS as a sensitivity analysis, and if the results of BMAS $\mathrm{sig}_{\text {are }}$ substantially different from those of BMAS then report the results of BMAS $\mathrm{sig}_{\text {sig }}$ as well (because this disparity in results may suggest a violation of assumptions of BMAS on publication bias); 
4. Use another prior than the default uniform prior if you have reasons to believe that a uniform prior is unlikely, but report the results of the uniform prior as well to show how much influence your prior has on your conclusions. 


\section{References}

American Psychological Association (2008). Reporting standards for research in psychology: Why do we need them? What might they be? The American Psychologist, 63(9), 839-851.

Augusteijn, H. E. M., van Aert, R. C. M., \& van Assen, M. A. L. M. (2019). The effect of publication bias on the Q test and assessment of heterogeneity. Psychological Methods, 24, 116134.

Borenstein, M., Hedges, L.V., Higgins, J.P.T., \& Rothstein, H.R. (2009). Introduction to metaanalysis. West Sussex: John Wiley \& Sons, ltd.

Bornmann, L., \& Mutz, R. (2015). Growth rates of modern science: A bibliometric analysis based on the number of publications and cited references. Journal of the Association for Information Science and Technology, 66(11), 2215-2222.

Carter, E. C., Schönbrodt, F. D., Gervais, W. M., \& Hilgard, J. (2019). Correcting for Bias in Psychology: A Comparison of Meta-Analytic Methods. Advances in Methods and Practices in Psychological Science, 2(2), 115-144. Doi: 10.1177/2515245919847196

Cohen, J. (1988). Statistical Power Analysis for the Behavioral Sciences. New York: Routledge, doi: $10.4324 / 9780203771587$

Cooper, H., Hedges, L. V., \& Valentine, J. C. (2009). The handbook of research synthesis and metaanalysis. New York, NY: Russell Sage Foundation.

Cooper, H., DeNeve, K., \& Charlton, K. (1997). Finding the missing science: The fate of studies submitted for review by a human subjects committee. Psychological Methods, 2(4), 447-452. doi:10.1037/1082-989X.2.4.447

Cordray, D. S., \& Morphy, P. (2009). Research synthesis and public policy. In H. Cooper, L. V. Hedges, \& J. C. Valentine (Eds.), The Handbook of Research Synthesis and Meta-Analysis (pp. 473-493). New York: Russell Sage Foundation.

Davey, J., Turner, R. M., Clarke, M. J., \& Higgins, J. P. (2011). Characteristics of meta-analyses and their component studies in the Cochrane Database of Systematic Reviews: a cross-sectional, descriptive analysis. BMC medical research methodology, 11(1), 1-11.DerSimonian, R., Laird, N. (1986). Meta-analysis in clinical trials. Controlled Clinical Trials, 7,177-188.

Dickersin, K. (2005). Publication bias: Recognizing the problem, understanding its origins and scope, and preventing harm. In H.R. Rothstein, A.J. Sutton \& M. Borenstein (Eds.), Publication bias in Meta-analysis, 11-33. New York: John Wiley \& Sons Inc. 
Duval, S., \& Tweedie, R. (2000). Trim and fill: a simple funnel-plot-based method of testing and adjusting for publication bias in meta-analysis. Biometrics, 56(2), 455-463.

Egger, M., Smith, G. D., Schneider, M., \& Minder, C. (1997). Bias in meta-analysis detected by a simple, graphical test. British Medical Journal, 315, 629-634.Fanelli, D. (2012). Negative results are disappearing from most disciplines and countries. Scientometrics, 90(3), 891-904. doi:10.1007/s11192-011-0494-7

Franco, A., Simonovits, G., \& Malhotra, N. (2016). Underreporting in psychology experiments: Evidence from a study registry. Social Psychological and Personality Science, 7(1), 8-12. doi:10.1177/1948550615598377

Friese, M., \& Frankenbach, J. (2020). p-Hacking and publication bias interact to distort metaanalytic effect size estimates. Psychological Methods, 25(4), 456-471. https://doi.org/10.1037/met0000246

Gelman, A. (2006). Prior distributions for variance parameters in hierarchical models (comment on article by Browne and Draper). Bayesian analysis, 1(3), 515-534.

Hedges, L. V. (1984). Estimation of effect size under nonrandom sampling: The effects of censoring studies yielding statistically insignificant mean differences. Journal of Educational Statistics, 9(1), 61-85.

Hedges, L.V., \& Vevea, J. (2005). Selection method approaches. In H.R. Rothstein, A.J. Sutton, \& M. Borenstein (Eds). Publication bias in meta-analysis: Prevention, assessment and adjustment (pp. 145-174). Chichester, England: John Wiley \& Sons, Ltd. doi:10.1002/0470870168.ch1

Higgins, J., \& Thompson, S. (2002). Quantifying heterogeneity in a meta-analysis. Statistics in medicine, 21, 1539-1558. doi:10.1002/sim.1186

Higgins, J. P., Thompson, S. G., Deeks, J. J., \& Altman, D. G. (2003). Measuring inconsistency in meta-analyses. Bmj, 327(7414), 557-560.

Huedo-Medina, T.B., Sánchez-Meca, J., Marín-Martínez, F., \& Botella, J. (2006). Assessing heterogeneity in meta-analyses: Q static or I2 index? Psychological methods, 11, 193-206.

IntHout, J., Ioannidis, J. P., \& Borm, G. F. (2014). The Hartung-Knapp-Sidik-Jonkman method for random effects meta-analysis is straightforward and considerably outperforms the standard DerSimonian-Laird method. BMC medical research methodology, 14(1), 25.

Jackson, D. (2006). The implications of publication bias for meta-analysis' other parameter.

Statistics in medicine, 25, 2911-1921. doi:10.1002/sim.2293 
Jackson, D. (2007). Assessing the implications of publication bias for two popular estimates of between-study variance in meta-analysis. Biometrics, 63, 187-193

Jeon, M., \& De Boeck, P. (2017). Decision qualities of Bayes factor and p value-based hypothesis testing. Psychological Methods, 22(2), 340.

Kass, R. E., \& Raftery, A. E. (1995). Bayes factors. Journal of the american statistical association, 90(430), 773-795.

Klein, R. A., Ratliff, K. A., Vianello, M., Adams Jr, R. B., Bahník, Š., Bernstein, M. J., ... \& Cemalcilar, Z. (2014). Investigating variation in replicability: A "many labs“ replication project. Social psychology, 45(3), 142-152. doi: 10.1027/1864-9335/a000178

Lane, D. M., \& Dunlap, W. P. (1978). Estimating effect size: Bias resulting from the significance criterion in editorial decisions. British Journal of Mathematical \& Statistical Psychology, 31, 107-112.

Langan, D., Higgins, J. P., \& Simmonds, M. (2017). Comparative performance of heterogeneity variance estimators in meta-analysis: a review of simulation studies. Research synthesis methods, $8(2), 181-198$.

Lin, L., \& Chu, H. (2018). Quantifying publication bias in meta-analysis. Biometrics, 74(3), 785794.

McCall, R. B., \& Carriger, M. S. (1993). A meta-analysis of infant habituation and recognition memory performance as predictors of later IQ. Child development, 64(1), 57-79.

McShane, B. B., Böckenholt, U., \& Hansen, K. T. (2016). Adjusting for publication bias in metaanalysis: An evaluation of selection methods and some cautionary notes. Perspectives on Psychological Science, 11, 730-749

Nuijten, M. B., van Assen, M. A. L. M., Veldkamp, C. L. S., \& Wicherts, J. M. (2015). The replication paradox: Combining studies can decrease accuracy of effect size estimate. Review of General Psychology, 19 (2), 172-182.

Olsson-Collentine, A., Wicherts, J. M., \& van Assen, M. A. L. M. (2020). Heterogeneity in direct replications in psychology and its association with effect size. Psychological Bulletin. Advance online publication. doi:10.1037/bul0000294

Schönbrodt, F. D., \& Wagenmakers, E. J. (2018). Bayes factor design analysis: Planning for compelling evidence. Psychonomic bulletin \& review, 25(1), 128-142.

Simonsohn, U., Nelson, L.D., \& Simmons, J.P. (2014). P-curve and effect size: Correcting for 
publication bias using only significant results. Perspectives on psychological science, 9, 666681. doi:10.1177/1745691614553988

Simonsohn, U., Simmons, J.P., \& Nelson, L.D. (2017). Datacolada [61] - Why p-curve excludes ps $>.05$. Retrieved from http://datacolada.org/61

Stanley, T. D. (2017). Limitations of PET-PEESE and other meta-analysis methods. Social Psychological \& Personality Science, 8, 581-591. doi:10.1177/1948550617693062

Stanley, T. D., \& Doucouliagos, H. (2014). Meta-regression approximations to reduce publication selection bias. Research Synthesis Methods, 5, 60-78.

Tendeiro, J. N., \& Kiers, H. A. (2019). A review of issues about null hypothesis Bayesian testing. Psychological methods, 24(6), 774-795. doi:10.1037/met0000221

Terrin, N., Schmid, C. H., Lau, J., \& Olkin, I. (2003). Adjusting for publication bias in the presence of heterogeneity. Statistics in Medicine, 22, 2113-2126.

van Aert, R. C. M., \& van Assen, M. A. L. M. (2021). Correcting for publication bias in a metaanalysis with the P-uniform* method. doi:10.31222/osf.io/zqjir9

van Aert, R. C. M., Wicherts, J. M., \& van Assen, M. A. L. M. (2016). Conducting meta-analyses based on p-values: Reservations and recommendations for applying $\mathrm{p}$-uniform and $\mathrm{p}$-curve. Perspectives on Psychological Science, 11, 713-729.

van Aert, R., Wicherts, J. M., \& van Assen, M. (2019). Publication bias examined in meta-analyses from psychology and medicine: A meta-meta-analysis. PloS one, 14(4), e0215052.

https://doi.org/10.1371/journal.pone.0215052

van Assen, M. A. L. M., van Aert, R. C. M., \& Wicherts, J. M. (2015). Meta-Analysis Using Effect Size Distributions of Only Statistically Significant Studies. Psychological Methods, 20(3), 293309. doi:10.1037/met0000025.

van Erp, S., Verhagen, J., Grasman, R. P., \& Wagenmakers, E. J. (2017). Estimates of between-study heterogeneity for 705 meta-analyses reported in Psychological Bulletin from 1990-2013. Journal of Open Psychology Data, 5(1). doi:10.5334/jopd.33

Veroniki, A. A., Jackson, D., Viechtbauer, W., Bender, R., Knapp, G., Kuss, O., \& Langan, D. (2015). Recommendations for quantifying the uncertainty in the summary intervention effect and estimating the between-study heterogeneity variance in random-effects meta-analysis. Cochrane Database Syst Rev, (Suppl 1), 25-27. 
Viechtbauer, W. (2007). Confidence intervals for the amount of heterogeneity in meta-analysis. Statistics in medicine, 26(1), 37-52.

Viechtbauer, W. (2010). Conducting meta-analyses in R with the metafor package. Journal of Statistical Software, 36(3), 1-48. doi:10.18637/jss.v036.i03Williams, D. R., Rast, P., \& Bürkner, P. C. (2018). Bayesian meta-analysis with weakly informative prior distributions. 10.31234/osf.io/7tbrm 